\title{
Estimating geostrophic and total velocities from CTD and ADCP data: intercomparison of different methods.
}

\author{
Anna Rubio $^{\mathrm{a},{ }^{*}, \text { Damià Gomis }}{ }^{\mathrm{b}}$, Gabriel Jordà ${ }^{\mathrm{b}}$, Manuel Espino ${ }^{\mathrm{a}}$ \\ ${ }^{a}$ Laboratori d'enginyeria maritima, LIM - UPC, Barcelona Spain \\ ${ }^{b}$ Institut Mediterrani d'Estudis Avançats, IMEDEA-CSIC, Esporles, Spain \\ * Fisrt autor current affiliation: Laboratoire de Physique des Océans, CNRS- \\ IFREMER-UBO-IRD, Brest, France
}

\begin{abstract}
Inferring geostrophic velocity fields from CTD data distributions can be handicapped by the impossibility of referring dynamic height to a no-motion level. This is often the case over the continental shelf, but also at open sea, even when velocity measurements (e.g. from a vessel mounted ADCP) are available. In this paper we test and compare four different methods aimed to estimate the geostrophic and total velocity fields from hydrodynamical data. Two of them can either use only CTD data (then relying on the election of a no-motion level) or incorporate ADCP data (through a multivariate interpolation); the other two methods always combine CTD and $\mathrm{ADCP}$ data. A $3 \mathrm{D}$ primitive equation model is used to reproduce realistic scenarios that provide control velocity fields and typical CTD and ADCP data profiles. The chosen scenarios represent different dynamic situations (in terms of data quality, bathymetric constrictions and dynamical characteristics such as the relative ageostrophic/geostrophic velocity variance) and make possible a broad discussion on the capabilities and limitations of the examined methods. Results show that the performance of the methods is highly dependent on the dynamics to be resolved. The combination of CTD and ADCP data constitutes the best approach for most of the analyzed situations, though special attention has to be paid when dealing with low quality $\mathrm{ADCP}$ data and when the circulation is characterized by intense non-divergent ageostrophic velocities.
\end{abstract}

KEYWORDS: Physical oceanography, geostrophic circulation; Spatial objective analysis, gradient wind balance, CTD data, ADCP data. 


\section{Introduction}

33 Inferring geostrophic velocities from CTD data distributions is reasonably simple. The most important limitation is that dynamic heights have to be referred to a given level surface, the so called "reference level". In the deep ocean the reference level is often taken as deep as possible, in order to approach a true no-motion level (hereafter NML) and hence to capture the whole baroclinic contribution to the motion field. However, the data availability at deep levels is sometimes constrained by the sampling methodology (e.g. when data is acquired by an undulating CTD) or by the bathymetry (e.g. when the surveyed domain includes the continental shelf). In such cases, the choice of a proper reference level is problematic: errors in the derived geostrophic velocity can be of the order of several $\mathrm{cm} / \mathrm{s}$, having a large impact on higher-order derived variables such as vorticity or the vertical component of the velocity.

Several methods have been developed to overcome these problems. A first group includes the methods that assume the existence of a NML. In coastal regions this level often intersects the bathymetry and therefore many CTD profiles may not extend down to the NML. In such cases the simplest approach is to compute dynamic height only at those stations reaching the reference level, but this usually results in large data voids over the continental shelf. Another common approach is to complete the missing lower part of the water column of shallow profiles (i.e., the layer in between the lower limit of the profile and the reference level) with the same layer of the nearest offshore station that does reach the NML. In practice this so-called nearest-neighbour approach has been applied in different ways.

Still within the same group of methods, some better founded approaches have been developed. For instance, the one proposed by Csanady (1979), aimed to estimate the along-shore and across-shore pressure gradients on the continental shelf. The theoretical frame of the method assumes that density gradients are parallel to bathymetry gradients and that the surface elevation field is induced by a uniform density along the coastline. These requirements are not fulfilled in regions where the circulation over the shelf shows significant along-shore gradients and eddies; in such cases Csanady's method is not more accurate than the simple methods described above. 
Another method proposed by Pedder and Gomis (1998), bases on the use of empirical

60 orthogonal functions. The leading modes of the vertical structure are first computed from

61 profiles spanning the whole vertical domain. Then, the amplitudes of these modes are computed for shallow stations under the constraint of reproducing the existing part of the profile. Finally,

63 the missing part of the profile is obtained by using the leading modes in its full vertical

64 extension. The main limitation of this method is the assumed statistical homogeneity between the shelf and offshore domains, an assumption that is not always fulfilled.

A second group of methods are those that do not necessarily assume the existence of a NML, but make use of independent velocity observations (e.g. obtained by a vessel mounted ADCP) to define a "level of known motion", rather than a NML. The two main methods falling within this group are the one proposed by Chereskin and Trunnell (1996) and the one proposed by Rudnick (1996). Both methods are constrained by the same assumption: that the non-divergent component of the measured velocity field is equivalent to the addition of the geostrophic velocity (due to the baroclinic component relative to a reference level) plus the eventual barotropic component of the flow. That is to say that the ageostrophic non-divergent component of the field is negligible compared with the geostrophic component.

Finally, another option to estimate the geostrophic velocity over the entire domain is the use of inverse models such as the beta-spiral method (Stommel and Schott, 1977; Schott and Stommel, 1978), the Wunsch method (Wunsch, 1978) or the Bernoulli method (Killworth, 1986). All of them rely on constraining the recovered hydrodynamical fields to obey some balance equations, typically geostrophy, hydrostatic balance and mass conservation (Davis, 1978). They are specially useful at open sea, typically for large-scale domains enclosed by observation transects but poorly sampled inside (Wunsch and Grant, 1982); in such cases, the constraints are reasonable and help to recover the poorly sampled field. However, in the case of small, well sampled coastal regions, the methods described above are more suitable, since some of the constraints used by inverse models (the mass conservation, for instance) may not apply. 
In this work, we test the capabilities and limitations of four of the methods described above: two methods based on the election of a NML [The methods proposed by Csanady (1979) and by Pedder and Gomis (1998) are not considered because their assumptions do not fit with the dynamics of the selected scenarios] and the methods proposed by Chereskin and Trunnell (1996) and Rudnick (1996) that combine CTD and ADCP data to infer geostrophic velocities. The methods are applied to synthetic data extracted from different realistic scenarios produced by a numerical model that intend to simulate different coastal and open sea regions of the western Mediterranean Sea. Density and velocity pseudo-observations are extracted simulating a real surveying strategy in terms of spatial sampling and observational errors.

Except for one of the examples, the pseudo-observations are not time dependent and thus they do not account for an eventual lack of synopticity of the data set. The lack of synopticity is recognized as a key error source (Gomis et al., 2005), but the magnitude of the associated errors is highly dependent of the dynamics of the region and on the survey strategy. Here we give some estimation of the magnitude of these errors relative the errors associated with the different diagnostic methods, but the nucleus of the work focuses on the intercomparison between the methods themselves. The application of all the methods to these pseudo-observations implies the use of an interpolation scheme, which is also recognized as an error source (Gomis and numerical model.

The paper is organized as follows. The main methodological aspects are presented in section 2: first, the different analysis methods and the interpolation scheme; next, the extraction of data from the numerical model; and last, the scenarios and test cases constituting the core of this work. In section 3 we present the results, dedicating a subsection to each test case. They are all discussed in section 4 and conclusions are outlined in section 5 . 


\section{2. Methodology}

\section{$114 \quad 2.1$ Analysis methods}

115 Within the group of methods that assume a NML, the approach of computing dynamic height 116 only for the profiles extending down to the reference level is referred to as 'standard NML'; this 117 is the first of the four methods considered in this work. In this approach, stations not reaching 118 the reference level are simply not considered in the computations, so that in the regions covered 119 by shallow profiles dynamic heights are extrapolated from deeper nearby stations during the 120 spatial objective analysis that precedes the computation of geostrophic magnitudes.

121 The second method is a version of the nearest-neighbour method: at shallow stations dynamic height is computed at each level relative to the next (in the sense of the vertical spacing of the output grid) and not relative to a common reference level. After station dynamic height data have been interpolated onto the grid, all levels are referred to the lowest one by adding the contributions of all the levels below. The a priori advantage of this method with respect to the standard NML method is that profiles obtained at shallow stations take part in the recovery of the dynamic height field (only the missing part of the water column is interpolated from nearby stations). This method will be referred to as 'stepped NML.'

The third method belongs to those based on a level of known motion and was proposed by

130 Chereskin and Trunnell (1996) (hereafter referred to as the 'Chereskin method'). This method consists of estimating dynamic height relative to a level $\mathrm{z}_{0}$ where the total velocity field can be determined from independent, simultaneous measurements. Following Chereskin and Trunnell (1996) at any level z we can recover the total geostrophic stream function $\left(\Psi_{g}\right)$ as the addition of two contributions: (i) the baroclinic stream function (i.e. the dynamic height) referred to the known motion level $z_{\mathrm{o}}\left(\left.\Psi_{\mathrm{g}}\right|_{z_{0}}\right)$, obtained from hydrographic data, and (ii) the stream function

136 obtained from the velocity field at $\mathrm{z}_{\mathrm{o}}\left(\Psi_{f}\left(\mathrm{z}_{0}\right)\right)$, which contains the baroclinic contribution of all levels underneath $\mathrm{z}_{0}$ plus an eventual barotropic component: 


$$
\Psi_{g}=\left.\Psi_{g}\right|_{z_{0}}+\Psi_{f}\left(\mathrm{z}_{0}\right)
$$

139 Although in principle $\mathrm{z}_{0}$ can be any depth at which we know the total velocity field, in practice 140 the election is constrained by two features. On one hand, it should be chosen as deep as possible 141 to avoid the presence of ageostrophic currents, which are usually stronger at surface layers. On 142 the other hand, the quality of ADCP data usually decreases with depth, and therefore the reference level should be chosen where the noise-to-signal ratio of velocity data is still acceptable. Another reason to avoid a deep reference level is that in the vicinity of the continental shelf the intersection with the bathymetry prevents the computation of the baroclinic stream function $\left.\Psi_{g}\right|_{z_{0}}$ at shallow stations.

In order to mix in a consistent way the geostrophic velocities obtained from CTD data and the velocity measurements at $\mathrm{z}_{0}$, only the non-divergent part of the latter is considered (expressed as $\Psi_{f}\left(\mathrm{z}_{0}\right)$ in [1]). The objective is to filter out ageostrophic components of the velocity field such as those produced by tidal or inertial oscillations. Hence, the method implicitly assumes that the non-divergent part of the velocity field measured at level $z_{\mathrm{o}}$ is equivalent to the total geostrophic velocity field (i.e., to the baroclinic component from a reference level up $z_{o}$ plus an eventual barotropic current). This equivalence is not always reasonable, as it depends on the case-to-case dynamics. For instance, a constant wind far from the coast can induce ageostrophic nondivergent currents within the Ekman layer, in which case the hypothesis will only be fulfilled below the Ekman layer. More problematic are deep, rapidly rotating vortices, which can have a significant non-divergent ageostrophic component extending beyond the lower limit of velocity measurements.

Finally, the fourth method is the one proposed by Rudnick (1996). This method is a generalization of the Chereskin method: it also bases on the comparison between the geostrophic stream function obtained from CTD data and the stream function derived from observed velocities. However, in Rudnick's method $\Psi_{f}\left(\mathrm{z}_{0}\right)$ is not estimated from the velocity field measured at $\mathrm{z}_{0}$, but from the differences between the dynamic height field computed from 
164 CTD data $\left.\Psi_{\mathrm{g}}\right|_{z_{0}}(\mathrm{z})$ and the stream function computed from the velocity measurements at the 165 same level $\Psi_{\mathrm{g}}(\mathrm{z})$. In principle this difference should be constant in depth, so that doing it at 166 any level $\mathrm{z}$ would be enough. In practice this is not the case, due to the incidental presence of 167 ageostrophic contributions (which can vary with depth) and to the impact of observational 168 errors. In order to minimize these undesirable contributions, Rudnick proposed to obtain $169 \Psi_{f}\left(\mathrm{z}_{0}\right)$ as the average of the differences $\left(\Psi_{g}-\left.\Psi_{g}\right|_{z_{0}}\right)$ obtained at the different levels, with the aim that non desired contributions average to zero. Once $\Psi_{f}\left(\mathrm{z}_{0}\right)$ is determined, the total geostrophic stream function can be obtained at every level following [1].

172 Note that if the average proposed by Rudnick really smoothes the impact of ageostrophic structures and observational errors on the analysis, some of the constraints on the election of the reference level mentioned for the Chereskin method can be relaxed. Still, the intersection with the bathymetry will prevent the computation of the geostrophic stream function at shallow stations, and therefore the level $z_{0}$ should not be too deep in the vicinity of the continental shelf. When applying his method in practice, Rudnick (1996) noted that the average of the differences is equivalent to the difference of the averages. Hence, $\Psi_{f}\left(\mathrm{z}_{0}\right)$ can be obtained as the difference between the stream function obtained from the vertically integrated total velocities $(\tilde{u}, \widetilde{v})$ and the vertically integrated dynamic height (equivalent to the stream function obtained from the vertically integrated baroclinic velocities computed relative to $z_{0},\left.\tilde{u}_{g}\right|_{z_{0}},\left.\widetilde{v}_{g}\right|_{z_{0}}$ ):

$$
\Psi_{f}\left(z_{0}\right)=\Psi(\tilde{u}, \tilde{v})-\Psi\left(\left.\tilde{u}_{g}\right|_{z_{0}},\left.\tilde{v}_{g}\right|_{z_{0}}\right)
$$

\subsection{Interpolation schemes}

The tests carried out for each method intend to simulate the actual processing of data from oceanographic surveys. Hence, the pseudo-observations extracted from model outputs are first interpolated onto a regular grid in order to compute derived magnitudes such as geostrophic velocities or vorticity. In order to facilitate the comparison with model values, the interpolation 
grid will be the same than the model grid (see Figures 1-3). However, it is worth noticing here that when the results of the different methods are displayed in Figures 4-8, only one out of two rows and columns are displayed, in order to simplify the Figures.

191 A common interpolation scheme is used for all the methods; it bases on the principles of 192 optimal statistical interpolation (see for instance Bretherton et al, 1976). This common scheme is the simplest version of OSI, and only considers observations of the variable to be interpolated (univariate approach, hereinafter referred to as "OSI UV"). Additionally, a second version of OSI is used for two of the methods: it is a multivariate version (referred to as "OSI MV") in which independent velocity data (e.g. ADCP data) are combined with dynamic height observations (e.g. obtained from CTD profiles) to produce the spatial interpolation of both fields

\section{8 (Gomis et al, 2001).}

The multivariate version bases on the physical relation linking the two variables and takes advantage that each field contains information on the other one. In fact, the velocity data entering the multivariate OSI scheme are not actual velocity observations, but the velocity shear with respect to the reference level used to compute dynamic height. If the subtracted velocity field (the velocity field at the reference level) is afterwards added to the analysis, the method becomes equivalent to the Chereskin method except in two features: i) dynamic height is obtained via multivariate analysis and therefore is supposed to benefit from a more accurate interpolation; ii) the multivariate interpolation produces two different estimations, one for the geostrophic velocity (derived from the reconstructed dynamic height) and one for the total velocity, so that the ageostrophic component of the field is also recovered (see Gomis et al., 2001).

210 The drawback of multivariate analysis is that it cannot extend below the vertical range of ADCP 211 data. The same happens with a direct interpolation of ADCP data (using either a univariate 212 analysis for each of the two velocity components or a multivariate version linking the two components). The latter will be referred to as OSI-ADCP and altogether with the multivariate interpolation must be considered at a different level than the four tested methods, since they 
215 cannot produce the current field below the ADCP domain (the other methods can provide

216 estimates for the whole vertical extent of hydrographic data).

217 The main characteristics of the interpolation schemes are given in the Appendix. The

218 parameters used to carry out the interpolation for each of the test cases described in section 2.4

219 are summarized in Table 1.

\subsection{Building-up the pseudo-observation data set}

221 The hydrographic and velocity data are obtained from numerical simulations of the circulation in the Mediterranean Sea. For that purpose, we use the 3D free-surface primitive equation model SYMPHONIE (Marsaleix et al., 1998; Marsaleix et al., 2008), which produces realistic scenarios including a wide variety of structures and processes. In particular, ageostrophic motion caused by current-topography interactions or wind forcing is included; conversely, tides are not included in the simulations. The model has a horizontal resolution of $3 \mathrm{~km}$ and 41 vertical hybrid $\sigma-z$ layers (Marsaleix et al., 1998). The control fields used to test the skill of the different methods are the geostrophic field (inferred from the model 3D density field and the free surface height) and the total velocity fields of the model outputs.

For each scenario we extract salinity, temperature and velocity profiles at given stations. The resulting data set is characterized by its vertical extent and by its horizontal resolution. In all the cases considered here, the station distribution consists of $8 \times 8$ equally spaced stations ( $12 \mathrm{~km}$ in each direction), with vertical profiles that reach a maximum depth of $1000 \mathrm{~m}$ for $\mathrm{S}$ and $\mathrm{T}$ (when not constricted by the bathymetry), and $350 \mathrm{~m}$ for the velocities (Table 1).

In order that the extracted temperature and salinity profiles have the characteristics of actual CTD profiles and velocities resemble those obtained by a vessel mounted ADCP, errors are added to the data extracted from the model. The salinity and temperature profiles are distorted by a vertically varying random error in the way: 


$$
\left[\begin{array}{c}
\mathrm{T}_{\mathrm{z}=0}^{\mathrm{obs}} \\
\mathrm{T}_{\mathrm{z}=1}^{\mathrm{obs}} \\
\cdot \\
\cdot \\
\mathrm{T}_{\mathrm{z}=z_{\max }}^{\mathrm{obs}}
\end{array}\right]=\left[\begin{array}{c}
\mathrm{T}_{\mathrm{z}=0}^{\bmod } \\
\mathrm{T}_{\mathrm{z}=1}^{\bmod } \\
\cdot \\
\cdot \\
\mathrm{T}_{\mathrm{z}=z_{\max }^{\bmod }}^{\bmod }
\end{array}\right]+\left[\begin{array}{c}
\mathrm{r}_{1} \\
\mathrm{r}_{2} \\
\cdot \\
\cdot \\
\mathrm{r}_{\mathrm{n}}
\end{array}\right] * \mathrm{E}_{\mathrm{T}}, \quad\left[\begin{array}{c}
\mathrm{S}_{\mathrm{z}=0}^{\mathrm{obs}} \\
\mathrm{S}_{\mathrm{z}=1}^{\mathrm{obs}} \\
\cdot \\
\cdot \\
\mathrm{S}_{\mathrm{z}=z_{\max }^{\mathrm{obs}}}^{\mathrm{m}}
\end{array}\right]=\left[\begin{array}{c}
\mathrm{S}_{\mathrm{z}=0}^{\bmod } \\
\mathrm{S}_{\mathrm{z}=1}^{\bmod } \\
\cdot \\
\cdot \\
\mathrm{S}_{\mathrm{z}=z_{\max }^{\bmod }}
\end{array}\right]+\left[\begin{array}{c}
\mathrm{r}_{1} \\
\mathrm{r}_{2} \\
\cdot \\
\cdot \\
\mathrm{r}_{\mathrm{n}}
\end{array}\right] * \mathrm{E}_{\mathrm{S}}
$$

240 where $r$ is a random number extracted from a zero-mean, unity-variance normal distribution, the

241 superindex 'obs' denotes the virtual observations and the superindex 'mod' denotes model control fields. $E_{T}$ and $E_{S}$ are the error standard deviations considered for temperature $(T)$ and salinity (S), respectively. For the velocity profiles, errors consist of a random contribution plus a vertically constant error that intends to resemble a systematic error (e.g. derived from the uncertainty in the navigation data used to subtract the ship velocity). This systematic error can be different for each profile (though the magnitude is considered the same):

where $\mathrm{r}$ and $\mathrm{b}$ are again random numbers extracted from a zero-mean, unity-variance normal distribution, $\mathrm{E}_{\mathrm{V}}^{\mathrm{rand}}$ is the $\mathrm{ADCP}$ error standard deviation and $\mathrm{E}_{\mathrm{V}}^{\text {bias }}$ represents the magnitude of the vertically coherent error.

Except for test case III, data are extracted from the respective 3D model fields at a single time step, and therefore the lack of synopticity affecting actual surveys is not considered. Test case III has been considered to illustrate the effect that these errors may have on the performance of

254 the different methodologies.

\subsection{Scenarios and test cases}

256 Three different scenarios (named as A, B and C) are used to perform five different tests

257 (enumerated from I to V). The details on the assumed observation errors, the vertical range of 
pseudo-observations and the interpolation parameters used in each test are summarized in Table 1.

The first scenario (A) represents a dynamical situation very similar to that observed by Rubio et al. (2005) in the Catalan Sea. In this scenario (Figure 1) the slope current flows over the 1000$2500 \mathrm{~m}$ isobaths with a mean speed of about $20 \mathrm{~cm} / \mathrm{s}$ at $20 \mathrm{~m}$ depth. An anticyclonic eddy is observed over the shelf break, also characterized by velocities of about $20 \mathrm{~cm} / \mathrm{s}$ at $20 \mathrm{~m}$ and extending down to $100 \mathrm{~m}$ depth. The circulation is mainly geostrophic and the spatial mean speed at $20 \mathrm{~m}$ is around $12 \mathrm{~cm} / \mathrm{s}$. The field has a non-negligible ageostrophic component (spatial mean speed around $3 \mathrm{~cm} / \mathrm{s}$ ) located in the central area of the domain and clearly associated with the anticyclonic eddy.

This scenario is the base for test cases I, II and III. Cases I and II differ in the magnitude of the assumed ADCP errors: test II assumes ADCP errors one order of magnitude higher than test I, in order to evaluate the influence of data quality on the results. In test case III ADCP errors are the same than in case I, but pseudo-observations are extracted using 5 days of model simulations (the time period derives from typical surveying speeds). The simulated nonsynoptic sampling starts at the north-west part of the domain and transects perpendicular to the coast are subsequently covered. Hence, a major difference between case III and the others is that errors due to the lack of synopticity of the sampling will add to CTD and ADCP errors. Namely, the south-westwards propagation of the anticyclone over the shelf break and the cross-shore migrations of the slope current during the sampling period induce large errors at the southwest part of the domain (i.e. the last region covered by the synthetic sampling) when compared to the total reference field corresponding to the initial time step (not shown).

For these three test cases, the reference level is taken at $200 \mathrm{~m}$ for the standard NML method, since a deeper NML would result in very large dynamic height data voids. For the stepped method the reference level has been set to $1000 \mathrm{~m}$, though the method is also tested taking the NML at $200 \mathrm{~m}$, with the aim of comparing the results with those of the standard approach. For the Chereskin method the known-motion level is set at $200 \mathrm{~m}$. The same reference level is used 
for the Rudnick method, for which the integration domain extends from surface to $350 \mathrm{~m}$ (a common ADCP data range). The interpolation parameters are the same for all cases except the velocity NTS ratio, which is set according to the different velocity errors considered in tests I and II.

Scenario B is located over the continental shelf of the Gulf of Lions (Figure 2). In this case the velocity field is highly influenced by strong NW winds and simulates a situation observed by Estournel et al (2003). In the south-western sector of the Gulf of Lions a strong south-eastwards current contours the coast. At $20 \mathrm{~m}$ depth, the mean speed of the coastal current is about 11 $\mathrm{cm} / \mathrm{s}$, while the maximum value reaches $38 \mathrm{~cm} / \mathrm{s}$. The geostrophic contribution shows part of the coastal current and a shallow cyclonic gyre of about $60 \mathrm{~km}$ diameter located in the middle of the domain. The ageostrophic contribution contains the other part of the coastal current and also shows a strong divergence in the middle of the domain. At $20 \mathrm{~m}$ depth the mean ageostrophic speed is around $11 \mathrm{~cm} / \mathrm{s}$, slightly stronger than the geostrophic mean speed that is around 9.8 $\mathrm{cm} / \mathrm{s}$. The ratio of the ageostrophic component relative to the geostrophic one decreases with depth, but for the shallow stations it remains significant down to the bottom.

300 Scenario B is the basis of test case IV, carried out with the objective of evaluating the performance of the different methods in presence of highly ageostrophic fields. It is worth noting that the reference level cannot be chosen deep enough to assure the absence of ageostrophic velocities. For the standard NML method, dynamic height is referred to $80 \mathrm{~m}$, again with the aim of avoiding large data voids. For the stepped method the reference level has been chosen at $200 \mathrm{~m}$ depth. The dynamic height computed for Chereskin and Rudnick methods is referred to $80 \mathrm{~m}$, and the vertical domain considered for the Rudnick method extends from surface to $200 \mathrm{~m}$.

Finally, scenario C (Figure 3) corresponds to a situation dominated by a large, intense eddy, similar to those observed in the Algerian sub-basin (Ruiz et al. 2001). The open-sea eddy is centred over the 1000-2000 m isobaths and has a vertical extension of $1000 \mathrm{~m}$. The anticyclonic circulation has mean velocities of $40 \mathrm{~cm} / \mathrm{s}$ and maximum velocities of $80 \mathrm{~cm} / \mathrm{s}$ at $20 \mathrm{~m}$, showing 
312 an eddy with a diameter of $100 \mathrm{~km}$. At $200 \mathrm{~m}$ the eddy shows the same diameter, with mean

313 velocities around $35 \mathrm{~cm} / \mathrm{s}$ and maximum velocities of $61 \mathrm{~cm} / \mathrm{s}$. The field is mainly geostrophic,

314 as it corresponds to a large, coherent-in-depth structure in absence of wind. Nevertheless, the

315 ageostrophic circulation has a mean speed of about $12 \mathrm{~cm} / \mathrm{s}$ at $20 \mathrm{~m}$ and shows an anticyclonic

316 pattern clearly associated with the normal acceleration inherent to the eddy; unlike for scenario

317 B, this acceleration is mostly non-divergent (see for instance Gomis et al, 2001, for a similar

318 case in the Alboran subbasin).

319 Test case $\mathrm{V}$, based on this scenario, intends to assess the performance of the different methods when the field presents a significant ageostrophic non-divergent contribution. In this case there vertical domain considered for the Rudnick method extends from surface to $350 \mathrm{~m}$ depth.

\section{Results}

328 For each test case we compare the velocity fields produced by the different methods with the geostrophic and total control velocities obtained from the model. Regarding the comparison with total velocities, it is worth stressing that, of the tested methods, only the multivariate analysis applied to NML methods can account for the divergent part of the velocity field. The univariate version of NML methods and the Chereskin and Rudnick methods only give the nondivergent component. On the other hand, since the reference-level velocity field has not been added when applying the multivariate analysis to NML methods (in order to avoid becoming practically equivalent to the Chereskin method), the crucial difference between those and the Chereskin and Rudnick methods is that the latter incorporate information from underneath the reference level (both the baroclinic component and an eventual barotropic component). The 
comparison of the results of the different methods with the control total velocity fields must be examined in the light of these considerations.

Since the interpolation and model grids are the same for all the tests, the error field is obtained simply as the difference between estimated and model velocities at grid points. RMS errors (computed as $<\left(\mathrm{u}-\mathrm{u}_{\bmod }\right)^{2}+\left(\mathrm{v}-\mathrm{v}_{\bmod }\right)^{2}>^{1 / 2}$, where $<.>$ denotes a spatial average) and relative RMS errors (RMS errors divided by the RMS of the respective control fields, computed as $<\mathrm{u}_{\bmod }{ }^{2}+$ $\mathrm{V}_{\bmod }{ }^{2}{ }^{1 / 2}$ ) are summarized in Table 2 for the five test cases. Relative RMS errors allow the comparison of the performances of the different methodologies through the different test cases. In order to simplify the comparison, all values and Figures refer to $20 \mathrm{~m}$ depth, as we expect the errors to be similar (in terms of relative RMS) at greater depths..

\subsection{Test case I: Weak ageostrophic contribution over the shelf break}

Figure 4 shows the velocity field recovered by each method. The smallest errors correspond to Chereskin and Rudnick methods, with respective RMS errors of 3.6 and $4.1 \mathrm{~cm} / \mathrm{s}$ (with respect to the model geostrophic velocity control field, Table 2). The errors associated with the NML methods are significantly larger (more than $7 \mathrm{~cm} / \mathrm{s}$ ) when the reference level is set to $200 \mathrm{~m}$. However, when the stepped method uses data down to $1000 \mathrm{~m}$ the errors reduce to $3.7 \mathrm{~cm} / \mathrm{s}$. It is worth reminding that choosing such a deep reference level for the standard NML would imply that only a few profiles would enter the interpolation, then resulting in very large errors in the shallow areas.

The explanation of the numbers given above makes clear when looking at Figure 4: Chereskin, Rudnick and the stepped method referred to $1000 \mathrm{~m}$ properly resolve the slope current, whereas the standard NML and the stepped method referred to $200 \mathrm{~m}$ do not. The error distribution of the standard NML (Figure 4a) clearly concentrates over the slope, with values of up to $10 \mathrm{~cm} / \mathrm{s}$.

When compared with the total velocity control fields, the relative performance of the methods is similar: the RMS is about $5 \mathrm{~cm} / \mathrm{s}$ for the Chereskin and Rudnick methods, as well as for the stepped method referred to $1000 \mathrm{~m}$; when the NML methods are referred to $200 \mathrm{~m}$ the RMS 
increases up to $8 \mathrm{~cm} / \mathrm{s}$. It is worth noting that the direct interpolation of ADCP data gives errors of the order of $2.2 \mathrm{~cm} / \mathrm{s}$, a small value compared to those obtained for the four methods. Hence, it turns out that although the ageostrophic component of the field is relatively small, its impact on the recovery of both, geostrophic and actual velocities is not negligible.

\subsection{Test case II: Weak ageostrophic contribution over the shelf break, low quality} 369 ADCP data.

370 The quality of ADCP data obviously does not influence the univariate NML methods, since velocity observations are not used in the computations. Conversely, the impact of low quality ADCP data on the multivariate application of NML methods is significant: errors are larger than those obtained in the former test case (Table 2), to the point that the multivariate approach becomes worse than the univariate one. This occurs despite the MV approach attenuates in part the impact of large ADCP errors by giving less weight to velocity observations when their quality is poorer (a higher NTS ratio value is used in this case for the ADCP, see Table 1). The impact of low quality ADCP data is higher in the case of Chereskin and Rudnick methods. The results of the Chereskin method, which base on the velocity field at a single level, are particularly bad (RMS error of $8.8 \mathrm{~cm} / \mathrm{s}$ ), in fact they are worse than for the standard NML method. The Rudnick method clearly benefits of some error cancellation during the vertical integration, giving similar results to those of the previous case in terms of relative and absolute errors. Compared to the rest of the methods Rudnick gives better results than the standard NML, but worse than the stepped method referred to $1000 \mathrm{~m}$.

Total velocities obtained from interpolated ADCP data are also significantly worse than in the previous case: RMS errors are about $6.3 \mathrm{~cm} / \mathrm{s}$, being in this case slightly better for the multivariate version. The errors derived from the direct multivariate interpolation of ADCP data are similar to those obtained for the Rudnick and the stepped methods (the latter referred to $1000 \mathrm{~m})$. For the rest of methodologies, the differences between the recovered velocity field and the total velocity control fields are higher (over $8 \mathrm{~cm} / \mathrm{s}$ ), though in terms of relative RMS errors they are comparable to those obtained when recovering the geostrophic fields. 

synoptic sampling.

In this case, the non synopticity of observations generate large errors at the southwest part of the domain which is the last area covered by the synthetic sampling (Figure 5). As a result, all methods behave significantly worse and the relative difference between them become shorter (Table 2). For the geostrophic velocities, the worst performance is obtained using the standard and the stepped methods (for both the univariate and multivariate versions) when referred to $200 \mathrm{~m}$. That is because, in addition to the synopticity errors, they cannot recover correctly the slope current. The best results for the geostrophic fields are obtained using Chereskin, Rudnick or the stepped method referred to deep data $(1000 \mathrm{~m})$. These three methods yield similar errors: about $8 \mathrm{~cm} / \mathrm{s}$. In the case of the Chereskin method, the errors are of the same order than those obtained for the previous case, while the Rudnick and the stepped NML methods are now clearly worse with relative errors around 0.7 . For total velocities the worst results (errors over $13 \mathrm{~cm} / \mathrm{s}$, relative errors of 0.8 ) are obtained when using the univariate optimal interpolation of ADCP data. A tentative explanation could be that the ageostrophic contribution has smaller time scales and therefore is more affected by the non synopticity of observations; nevertheless, errors are reduced when using the multivariate version). As for the geostrophic component, the best results are obtained using Rudnick, Chereskin and the stepped method referred to $1000 \mathrm{~m}$.

\section{3.4 Test case IV: Large ageostrophic contribution over the shelf}

413 For the geostrophic velocities, the results of the different methods are quite close to each other, 414 but the analysis of the differences is still interesting. The worst results (errors over $6.7 \mathrm{~cm} / \mathrm{s}$ ) 415 correspond to Chereskin and Rudnick, as well as to the univariate standard NML method. This 416 indicates that the velocities entering the Chereskin and Rudnick methods are contaminated by a 
417 significant ageostrophic contribution, even if only the non-divergent part is considered. The bad 418 results of the standard NML level can be attributed to the shallow reference level. The 419 multivariate application of the NML methods seems to improve the univariate one (for both

420 NML methods). This indicates that the multivariate analysis is successful in separating the 421 divergent and no-divergent part of the flow, which is one of the characteristics of the version of 422 the method applied here (for more details see Gomis et al., 2001). In the multivariate analysis, 423 only the non-divergent part is used to recover the geostrophic component.

424 The best estimation of the geostrophic field is produced by the multivariate stepped method with errors of about $5 \mathrm{~cm} / \mathrm{s}$ (Table 2 and Figure 6). Again, a key difference with respect to the methods except the stepped MV analysis have problems to recover the geostrophic velocity field. Errors are larger on the western side of the domain, where ageostrophic velocities are not the largest ones, but the bathymetry is very shallow (see Figure 2). For the total velocities all methods give estimations with comparable errors; they are all larger than $11 \mathrm{~cm} / \mathrm{s}$, which results in relative errors over 0.8 (i.e., notably larger than those obtained for the geostrophic fields). As it could be expect from a highly ageostrophic field, good estimations are only obtained from the direct interpolation of ADCP data, which gives RMS errors under 4 $\mathrm{cm} / \mathrm{s}$.

\subsection{Test case V: Energetic eddy at open sea.}

As expected, errors are very large (above $25 \mathrm{~cm} / \mathrm{s}$ in terms of absolute error, and 0.8 in terms of relative error of 0.8$)$ when a shallow reference level is chosen $(200 \mathrm{~m})$. When the deepest available level $(1000 \mathrm{~m})$ is chosen as reference level the errors decrease, but they are still quite

440 high (over $17 \mathrm{~cm} / \mathrm{s}$, see Table 2). This indicates either that the baroclinic contribution below $4411000 \mathrm{~m}$ is still significant or the presence of a significant barotropic component (at $1000 \mathrm{~m}$ the 442 model velocities are still of the order of $10 \mathrm{~cm} / \mathrm{s}$ ). 
The best estimations of the geostrophic field are by far produced by the Chereskin and Rudnick methods (around 5-6 cm/s RMS). Nevertheless, Figures 7c and 7d still show an error field with a clear anticyclonic pattern and speed errors around $10 \mathrm{~cm} / \mathrm{s}$. That is, both methods tend to overestimate the geostrophic component of the anticyclonic eddy. In fact, a feature that may be surprising at first sight is that both methods give a better estimate for the total velocities (errors between 4 and $5 \mathrm{~cm} / \mathrm{s}$ ) than for the geostrophic velocities. As it will be explained later on, the reason is that the geostrophic approximation fails to reproduce rapidly rotating vortices such as the one modelled in this case.

\section{Discussion}

Taken altogether, results suggest that the election of a method to estimate geostrophic and total velocity fields from in-situ observations is not a trivial question. However, there are some features that are common to all test cases and shed some light in the election process.

Concerning the estimation of geostrophic fields, Chereskin, Rudnick and the stepped NML have demonstrated similar skills, with some particularities that depend on the test case. Generally speaking, the three methods are able to estimate the geostrophic circulation with smaller errors than the standard NML, which is seriously handicapped by the topography. However, this is only true when the stepped NML can be applied to deep CTD data: if hydrographic data are not available down to a reasonable no-motion level, then Chereskin and Rudnick are the only methods than can recover the deep baroclinic contribution.

Chereskin and Rudnick are generally better than the stepped NML when the total velocity is to be recovered. The reason is that both methods can recover (at least to some extent) the barotropic component, whereas the stepped NML can at most recover the whole baroclinic component, but not the barotropic one. None of the methods is as accurate as the direct interpolation of ADCP data. [However, as stated above, the direct interpolation can only attempt to recover the velocity field within the ADCP range, whereas the other methods give results down to the deepest level covered by hydrographic observations.] 
Another common feature is that the multivariate version is not much better than the univariate one (i.e., the differences in favour of the first are small compared to the differences between the tested methods). On the positive side, multivariate OSI does not suffer too much in presence of high ADCP errors, provided that the NTS parameter is set accordingly to observational errors. Hence, beyond the described general features, the election of a particular method will depend on the characteristics of the particular case study.

The results leading to the general conclusions outlined above base on the assumption that ADCP velocities can be recovered with an uncertainty of the order of $1 \mathrm{~cm} / \mathrm{s}$ (partitioned in a random plus a systematic part). In practice, such accuracy can be achieved only if ADCP data are acquired and processed very carefully. If this is not the case, random errors can increase up to several $\mathrm{cm} / \mathrm{s}$; moreover, in absence of bottom-tracking the uncertainty of ship navigation data can also increase systematic errors by some $\mathrm{cm} / \mathrm{s}$. Test case II has shown that in such cases the performance of the Chereskin method is significantly poorer than the stepped NML method (which does not include ADCP data in the analysis), whereas the Rudnick method is much less handicapped. The reason is that at least the random part of the errors is partly averaged out during the vertical integration carried out by the Rudnick method. Conversely, Chereskin entirely relies on the accuracy of the velocity field at the level of known motion, and the errors involved in the estimation of the stream function at that level are added to the estimation of the geostrophic field at all depths.

Another assumption implicit in the above conclusions is that observations were synoptic. In the case of large and non random observational errors such as those derived from a non synoptic sampling (test case III) all methods result in significant errors when inferring the geostrophic fields. In this case Chereskin and Rudnick methods have similar performances to the stepped method applied to deep data (i.e., the vertical integration involved in Rudnick's method does not improve results significantly). This conclusions must however be taken with caution, since the impact of the lack of synopticity is strongly case dependent. For some surveys this impact can 
be small enough as for the above conclusions to be entirely valid, and for other surveys the impact can be much larger than the differences between the skills of the tested methods.

The conclusions stated above also hold for the estimation of total velocities. It is worth noting that in presence of poor quality ADCP data the stepped method is even better than the direct interpolation of ADCP data. The errors associated with the Rudnick method are of the same order than those associated with the direct interpolation. Another point to note is that the impact of the lack of sinopticity is probably larger for the ageostrophic component of the circulation (due to its smaller time scale) which strongly affects the direct interpolation of the ADCP data; nevertheless, these errors can be partly reduced by the use of CTD data through a multivariate approach. The best recovery of total velocities undertaken from a non synoptic sampling is obtained applying the stepped method to deep data or using Chereskin and Rudnick methods.

When the field contains large ageostrophic velocities (test case IV), the assumption that the nondivergent and the geostrophic fields are equivalent is no longer true (the ageostrophic component usually has a non-divergent part). In that case the addition of that component to the baroclinic one distorts the geostrophic velocity, rather than improving it. This explains why in such cases the stepped NML method (and even the standard NML) gives the best results for the geostrophic velocities. Another worth noting feature is that in the tested cases the use of ADCP data (even if they are "contaminated" by the non divergent ageostrophic contribution) produces a geostrophic field that is more energetic and, in consequence, closer to actual velocities. However, differences are small and it is difficult to assure that these results can extend to most ageostrophic situations.

In the case of the Chereskin method, the presence of a significant non-divergent ageostrophic contribution in the velocity field only affects the level of known motion, and therefore its impact can be minimized when the reference level is below the layer submitted to ageostrophic forcing. Conversely, in the Rudnick method the presence of a non-divergent ageostrophic contribution affects all levels in a systematic way (i.e., this component will likely not average out during the vertical integration) and therefore its impact cannot be minimized. In the 
particular test case IV, Chereskin and Rudnick give similar results because in Chereskin the level of known motion cannot be chosen very deep due to bathymetric constraints.

Regarding total velocities, it seems clear that in presence of a large ageostrophic component none of the methods can properly recover the actual field. For all of them, errors are significantly larger than for the direct interpolation of ADCP data.

Finally, test case V has examined a case without wind induced circulation, but with a deep, rapidly rotating structure (the structure of test case I also has strong curvature, but it is less intense and vanishes at a relatively shallow depth). The effect of rotation is that the normal acceleration term is no longer negligible and therefore the so-called gradient-wind balance

The fact that NML methods give much worse results than Chereskin and Rudnick is due to the (between the Coriolis, baric and normal accelerations) represents more accurately the total component of the velocity field than the geostrophic balance. For Rossby numbers $\mathrm{Ro}<<1$ the normal acceleration is negligible relative to the Coriolis and baric ones. For Ro 0.1-0.15 (test case I) the resulting non-divergent ageostrophic component is around $10-15 \%$ of the total velocity. This results in velocity errors of the order of $2-3 \mathrm{~cm} / \mathrm{s}$ at $20 \mathrm{~m}$, i.e., within the order of instrumental errors and therefore their impact is not expected to severely distort the results. For test case $\mathrm{V}$, however, the Rossby number is around $0.15-0.2$ and total velocities are of the order of $40 \mathrm{~cm} / \mathrm{s}$ at $20 \mathrm{~m}$. Hence, errors inherent to the geostrophic assumption are around $6-8 \mathrm{~cm} / \mathrm{s}$ at that level. The error fields of Figure 7 show that errors are clearly associated with the eddy and, as expected, they form a clockwise rotating closed pattern. vertical extension of the structure, which still has significant velocities at $1000 \mathrm{~m}$. Moreover, since the Chereskin and Rudnick methods include the non-divergent ageostrophic component (not included in NML methods), they also produce the best estimate for the total velocity field.

The non-divergent ageostrophic component cannot be avoided by the Chereskin method because the eddy is deeper than the ADCP range. Conversely, all methods can benefit from the application of the gradient-wind balance instead of the geostrophic balance. In particular, the Chereskin and Rudnick methods can be improved if the stream function $\Psi_{f}$ of [1] is not 
computed from the geostrophic balance but from the gradient wind balance. Using a natural coordinate system (Holton, 1992) the gradient wind balance can be written as:

$$
\frac{V_{g w}^{2}}{R}+f V_{g w}=-\frac{\partial \Phi}{\partial n}
$$

where $\mathrm{V}_{\mathrm{gw}}$ is the gradient wind velocity, $\mathrm{f}$ is the Coriolis parameter, $\mathrm{R}$ is the curvature radius and $\Phi$ is the streamfunction, so that the baric acceleration is given by $-\delta \Phi / \delta \mathrm{n}$. When R tends to $\pm \infty$ (i.e. when there is no curvature and in consequence the normal accelerations is negligible) expression [5] reduces to the geostrophic balance:

$$
f V_{g}=-\frac{\partial \Phi}{\partial n}
$$

And combining equations [5] and [6] it results that:

$$
\frac{V_{g w}^{2}}{R}+f V_{g w}=f V_{g}
$$

Following Shearman et al. (2000) the gradient wind velocities can be easily estimated from CTD observations after a few weak approximations. One of the simplifications proposed by Shearman et al. (2000) is the "Quasi geostrophic approximation", which consists of computing the curvature radius $\mathrm{R}$ and then the whole normal acceleration term from the geostrophic velocity derived from CTD observations. In this case, expression [7] becomes:

$$
V_{g w}=V_{g}-\frac{V_{g}^{2}}{f R}
$$

When simultaneous ADCP data are available, the curvature radius $\mathrm{R}$ and the whole normal acceleration can be inferred form the non divergent component of the ADCP velocity field:

$$
V_{g w}=V_{g}-\frac{V_{a d c p}^{2}}{f R}
$$

Taking for instance the second approach, the Chereskin and Rudnick forcing terms can be rewritten as:

$$
\Psi_{f}=\Psi_{a d c p}-\Psi_{g}+\Psi_{a d c p} \frac{\left|\Psi_{a d c p}\right|}{f R}
$$


572 where $\Psi_{\text {adcp }}$ and $\Psi_{\mathrm{g}}$ denote the stream functions derived from ADCP data and from dynamic 573 height, respectively (estimated at the reference level in the case of the Chereskin method and 574 vertically integrated through the whole domain in the Rudnick method).

575 In order to quantify the eventual benefits of this new approximation, we modified the Rudnick 576 method following [9] and used it to recalculate geostrophic and total fields for test case V

577 (Figure 8). Errors are significantly lower for the geostrophic field (around $3.1 \mathrm{~cm} / \mathrm{s}$ instead of $5.6 \mathrm{~cm} / \mathrm{s}$ obtained by using the classical scheme). The anticyclonic pattern observed in the error field of Figure 7 is no longer observed in Figure 8, since the contribution of the normal acceleration has now been considered. For the total velocities the new approximation yields higher errors $(7.1 \mathrm{~cm} / \mathrm{s})$ than the classical version of the Rudnick method $(3.9 \mathrm{~cm} / \mathrm{s})$. This is an expected result, since the main ageostrophic contribution to the total velocity field has now been removed from the estimations.

\section{Conclusions}

586 The main conclusion is that none of the methods is clearly superior to the others for all the tested scenarios. Nevertheless, some meaningful recommendations can be extracted from the results presented above:

1) In absence of large errors due to the lack of synopticity of the sampling and at those levels where ADCP data are available, a multivariate interpolation carried out in the way proposed by Gomis et al. (2001) is clearly the best option to recover the total velocity field. This method is quite robust in front of high ADCP errors, provided a right value is used on input for the noiseto-signal parameter. However, this method cannot be applied below the range of ADCP data

594 (typically 300 to $600 \mathrm{~m}$ depth), and therefore must be considered at a different level than the

595 four methods tested in this work. For non synoptic sampling, the use of deep CTD data or the combination of CTD data with ADCP data give significantly better results. 
597 2) For the recovery of the velocity field over the whole vertical range covered by CTD data, the 598 use of ADCP data collected at upper levels is the best option in most cases. The equivalence 599 between the geostrophic and the non-divergent total stream functions assumed by the Chereskin 600 and Rudnick methods gives the best estimates of the geostrophic and total fields for most 601 surveyed fields. However, this is not the case when:

602 (i) The quality of ADCP data is low. The Chereskin method is particularly sensitive to ADCP 603 errors, since the reference velocity field added to all levels relies on ADCP data acquired at a 604 single level. The Rudnick method is less sensitive, since it benefits from random error cancellations during the vertical integration. Nevertheless, systematic errors (those extending

606 through the whole profile) persist, and results indicate that when these are of the order of 10 $607 \mathrm{~cm} / \mathrm{s}$ it would be better to use only CTD data (e.g., in the way proposed by the stepped method).

608 (ii) The field is highly ageostrophic. In that case it will also be better to rely only on CTD data 609 to estimate the geostrophic component. None of the methods is able to recover the total velocity 610 with an acceptable accuracy.

611 iii) In presence of strong curvatures. In these cases, the use of the gradient wind balance is 612 strongly recommended for the forcing terms of Cherekin and Rudnick methods.

613 3) Over the continental shelf and when only CTD data are available, the stepped NML is clearly 614 superior to the standard NML method. The reason is simple: it can be applied to deep data 615 without resulting in large data voids in the dynamic height field. 


\section{Appendix: the Optimal Statistical Interpolation scheme}

618 The OSI scheme is linear: gridpoint values are obtained as a weighted sum of observations. The 619 main difference with respect to empirical methods (e.g. those based on distance-weighted 620 functions) is that OSI weight functions are determined under the constraint of minimizing (in a 621 statistical sense) the differences between the true field and the analysis. A related consequence 622 is that OSI not only considers the distance between each station and the grid points, but also the 623 distance of the stations relative to each other (see for instance Bretherton et al., 1976; Gomis et 624 al, 2001). The application of the OSI scheme makes necessary to fix some parameters. First, the values to be interpolated must have a zero mean: the scheme does not use raw observations, but the deviation between these and a statistical mean field (e.g., a climatology). In principle the mean field should be derived from historical data. However, since oceanographic data bases are too poor for this purpose, it is commonly assumed that the mean field can be obtained by fitting a low-order polynomial to observations. Thus, a first parameter to be fixed is the order of this polynomial (usually a low order, to avoid pouring most of the variability of the observed field into the mean field).

Other parameters to be determined are those characterizing the lag-correlation function, which is related to the scale of the structures dominating the dynamics of the surveyed domain. Here the correlation is assumed to be homogeneous and isotropic and to have a Gaussian shape, so that a single parameter (the so-called "characteristic scale length") is enough to determine the lag-correlation model.

A third parameter required by the OSI scheme refers to observational errors, here defined as a noise-to-signal ratio (hereafter NTS) parameter. This ratio is defined as the variance of the observational error divided by the variance of the interpolated field (the latter referring to the deviations between observations and the mean field). In the univariate version of OSI this parameter has been taken constant for all stations entering the 2D interpolations (though varying with depth). In the multivariate version of OSI the NTS parameters are different for dynamic 
644 height and velocity observations, and determine the influence of each variable on the analysis

645 (the largest the NTS parameter, the smallest the influence).

646 Finally, we also set a smoothing length scale, which is not strictly part of the OSI scheme, but it

647 has been shown to mitigate the aliasing produced by small structures that cannot be resolved by 648 the sampling (Gomis and Pedder, 2005). The smallest scale that can be resolved is determined 649 by the Nyquist wavelength, which is given by twice the mean spacing between stations. The 650 parameters used to carry out the interpolation for each of the test cases described in section 2.4 651 are summarized in Table 1.

652

\section{Acknowledgements}

656 This work has been partially performed in the framework of the project «Génesis, evolución temporal, translación y efectos de remolinos de mesoescala sobre la plataforma continental catalana» (MAR1999-1010), funded by the Spanish Marine Science and Technology Program, and the project "Mediterranean ocean Forecasting System: Toward environmental predictions" (MFSTEP, funded by the European Commission, under contract EVK3-CT-2002-00075). The works of A. Rubio and G. Jordà were funded by a grant from the Spanish Ministerio de Educación, Cultura y Deportes and by a grant of the Universitat Politècnica de Catalunya. The authors would like to thank the two anonymous reviewers whose comments helped to improve

664 the quality of this work. Finally, the authors also want to thank PRECARIOS, a postgraduate organisation whose dedication and efforts contribute to improving the working conditions for young scientists in Spain (www. precarios.org). 


\section{REFERENCES}

Bretherton F. P., R. E. Davis \& C. B. Fandry (1976). A technique for objective analysis and design of oceanographic experiments applied to MODE-73. Deep-Sea Research I, 23, $559-582$.

Chereskin and Trunnell, T.K. \& Trunnell, M. (1996). Correlation scales, objective mapping and geostrophic flow in the California current. Journal of Geophysical Research, 101 (C10), 22.619-22.629.

Csanady, G. T. (1979). The pressure field along the western margin of the North Atlantic. Journal of Geophysical Research, 81(C8), 4905, 4915.

Estournel, C., Durrieu de Madron, X., Marsaleix, P., Auclair, F., Julliand, C. \& Vehil, R. (2003). Observation and modelisation of the winter coastal oceanic circulation in the Gulf of Lion under wind conditions influenced by the continental orography (FETCH experiment). Journal of Geophysical Research, 108 (C3).

Gomis, D., Ruiz, S. \& Pedder, M.A. (2001). Diagnostic analysis of the 3D ageostrophic circulation from a multivariate spatial interpolation of CTD and ADCP data. Deep-Sea Research I, 48, 269-295.

Gomis, D. \& M. A. Pedder (2005). Errors in dynamical fields inferred from synoptic oceanographic cruise data. Part I: the impact of observation errors and the sampling distribution. Journal of Marine Systems, 56/3-4, 317-333.

Gomis, D., M. A. Pedder \& A. Pascual (2005). Errors in dynamical fields inferred from synoptic oceanographic cruise data. Part II: the impact of the lack of synopticity. Journal of Marine Systems, 56/3-4, 334-351.

Marsaleix P., Auclair F., Floor J. W., Herrmann M. J., Estournel C., Pairaud I., Ulses C. (2008). Energy conservation issues in sigma-coordinate free-surface ocean models. Ocean Modelling, 20 (1), 61-89.

Marsaleix, P., Estournel, C., Kondrachoff, V. \& Vehil, R. (1998). A numerical study of the formation of the Rhône river plume. Journal of Marine Systems, 14, 99-115.

Pedder, M. A. \& D. Gomis (1998). Application of EOF análisis to the spatial estimation of ocean circulation features sampled by high-resolution CTD casts. Journal of Atmospheric and Ocean Technology, 15, 959-978.

Rubio A., Arnau P.A., Espino, M., Flexas, M., Jordà, G., Salat, J., Puigdefàbregas, J. \& S.Arcilla, A. (2005). A field study of the behaviour of an anticyclonic eddy on the Catalan continental shelf (NW Mediterranean). Progress in Oceanography, 66, 2-4, 142-156.

Rudnick, D.L.(1996) Intensive surveys of the Azores front, 2. Inferring the geostrophic and vertical velocity fields. Journal of Geophysical Research, 101 (C7), 16.291-16.303.

Ruiz, S. (2000). Análisis espacial objetivo de datos oceanográficos: aplicaciones en el mar de Alborán. PhD Thesis. UPC-CSIC-UB. Barcelona.

Ruiz, S., Font, J., Emelianov, M., Isern-Fontanet, J., Millot, C., Salas, J., Taupier-Letage, I. (2002). Deep structure of an open sea eddy in the Algerian Basin. Journal of Marine Systems, 33-34, 179-195.

Shearman, K., J. A. Barth, J. S. Allen, R. L. Haney (2000). Diagnosis of the three-dimensional circulation in mesoscale features with large Rossby number". Journal of Physical Oceanography, 30, 2687-2709. 


\section{FIGURE CAPTIONS}

FIGURE 1: Snapshots of the (a) total, (b) geostrophic and (c) ageostrophic model velocity control fields at $20 \mathrm{~m}$ used for SCENARIO A (test cases I , II and III). X-axis and Y-axis: longitudes and latitudes in degrees.

FIGURE 2: Snapshots of the (a) total, (b) geostrophic and (c) ageostrophic model velocity control fields at 20m used for SCENARIO B (test case IV). X-axis and Y-axis: longitudes and latitudes in degrees.

FIGURE 3: Snapshots of the (a) total, (b) geostrophic and (c) ageostrophic model velocity control fields at $20 \mathrm{~m}$ used for SCENARIO C (test case V). X-axis and Y-axis: longitudes and latitudes in degrees.

FIGURE 4: From left to right: estimated geostrophic field, error field (with respect to the control geostrophic velocity) and module of the error field at $20 \mathrm{~m}$ obtained for TEST CASE I using the following methods: (a) Multivariate fixed NML, (b) Univariate stepped NLM referred to 1000 m, (c) Chereskin and (d) Rudnick. Xaxis and Y-axis: longitudes and latitudes in degrees.

FIGURE 5: From left to right: estimated geostrophic field, error field (with respect to the control geostrophic velocity) and module of the error field at $20 \mathrm{~m}$ obtained for TEST CASE III using the following methods: (a) Multivariate fixed NML, (b) Univariate stepped NLM referred to 1000 m, (c) Chereskin and (d) Rudnick. Xaxis and Y-axis: longitudes and latitudes in degrees.

FIGURE 6: From left to right: estimated geostrophic field, error field (with respect to the control geostrophic velocity) and module of the error field at $20 \mathrm{~m}$ obtained for TEST CASE IV using the following methods: (a) Multivariate fixed NML, (b) Univariate stepped NLM referred to $200 \mathrm{~m}$, (c) Chereskin and (d) Rudnick. X-axis and $\mathrm{Y}$-axis: longitudes and latitudes in degrees.

FIGURE 7: From left to right: estimated geostrophic field, error field (with respect to the control geostrophic velocity) and module of the error field at $20 \mathrm{~m}$ obtained for TEST CASE V using the following methods: (a) Multivariate fixed NML, (b) Univariate stepped NLM referred to 1000 m, (c) Chereskin and (d) Rudnick. Xaxis and Y-axis: longitudes and latitudes in degrees.

FIGURE 8: From left to right: estimated geostrophic field, error field (with respect to the control geostrophic velocity) and module of the error field at $20 \mathrm{~m}$ obtained using the Rudnick method corrected for the gradient wind balance in TEST CASE V velocity fields. $\mathrm{X}$-axis and $\mathrm{Y}$-axis: longitudes and latitudes in degrees. 
747

748

749

750

751

752

753

754

755

756

757

758

759

TABLE 1: Parameters used for the optimal interpolation carried out for each test case. From left to right: Test case and scenario, maximum vertical extent of the profiles and errors added to the synthetic profiles (ET, ES for the 'CTD data' and ADCP ERAND, EBIAS for the 'ADCP data'), noise to signal ratios (NTS), lag-correlation scale $(\mathrm{SCL})$ and cut-off wavelength $(\lambda)$.

TABLE 2: RMS differences $(\mathrm{cm} / \mathrm{s})$ between the estimated and the control velocity fields obtained for the different methods and each test case. RMS errors are estimated at $20 \mathrm{~m}$ depth, as explained in section 3. Relative RMS errors are calculated relative to the RMS of the geostrophic and total control fields at 20m depth. OSI ADCP denotes the direct interpolation of ADCP data using the Optimal Interpolation Scheme defined in the Appendix. UV stands for univariate and MV stands for multivariate and refers to the version of the OSI used to interpolate ADCP or CTD observations. 


\begin{tabular}{|c|c|c|c|c|c|c|c|c|}
\hline \multirow[b]{2}{*}{ 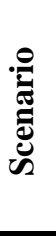 } & \multirow{2}{*}{ 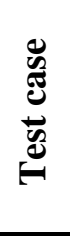 } & \multicolumn{2}{|c|}{ CTD data } & \multicolumn{2}{|c|}{ ADCP data } & \multicolumn{3}{|c|}{ Analysis } \\
\hline & & $\begin{array}{c}\text { Max. } \\
\text { Data } \\
\text { depth } \\
\text { (m) }\end{array}$ & $\begin{array}{c}\mathbf{E}_{\mathbf{T}}\left({ }^{\circ} \mathbf{C}\right), \\
\mathbf{E}_{\mathbf{S}}(\text { p.s.u })\end{array}$ & $\begin{array}{c}\text { Max. } \\
\text { Data } \\
\text { depth } \\
(\mathbf{m})\end{array}$ & $\begin{array}{c}\mathbf{E}_{\text {RAND }}(\mathrm{cm} / \mathrm{s}), \\
\mathbf{E}_{\text {BIAS }}(\mathrm{cm} / \mathrm{s})\end{array}$ & $\begin{array}{c}\text { NTS ratios } \\
\text { (CTD, ADCP) }\end{array}$ & $\begin{array}{l}\text { SCL } \\
(\mathbf{K m})\end{array}$ & $\lambda(\mathbf{K m})$ \\
\hline A & $\begin{array}{l}\text { I, } \\
\text { III }\end{array}$ & 1000 & $0.005,0.005$ & 350 & $0.5,0.5$ & $0.005,0.05$ & 15 & 20 \\
\hline A & II & 1000 & $0.005,0.005$ & 350 & 5,5 & $0.005,0.10$ & 15 & 20 \\
\hline $\mathrm{B}$ & IV & 350 & $0.005,0.005$ & 350 & $0.5,0.5$ & $0.005,0.05$ & 15 & 20 \\
\hline $\mathrm{C}$ & $\mathbf{V}$ & 1000 & $0.005,0.005$ & 350 & $0.5,0.5$ & $0.005,0.05$ & 15 & 20 \\
\hline
\end{tabular}

TABLE 1 


\begin{tabular}{|c|c|c|c|c|c|c|c|}
\hline & \multirow{2}{*}{\multicolumn{2}{|c|}{ Method }} & \multirow{3}{*}{$\begin{array}{c}\text { Ref level (m) } \\
- \\
\end{array}$} & \multicolumn{2}{|c|}{ VS. model geostrophic velocities } & \multicolumn{2}{|c|}{ VS. model total velocities } \\
\hline & & & & $\begin{array}{l}\text { RMS error } \\
\quad(\mathbf{c m} / \mathbf{s})\end{array}$ & $\begin{array}{l}\text { RMS error/ RMS } \\
\text { geostrophic control } \\
\text { field }\end{array}$ & $\begin{array}{l}\text { RMS error } \\
(\mathbf{c m} / \mathbf{s})\end{array}$ & $\begin{array}{l}\text { RMS error/ } \\
\text { RMS total } \\
\text { control field }\end{array}$ \\
\hline \multirow{8}{*}{ 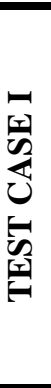 } & \multirow{2}{*}{$\begin{array}{l}\text { OSI- } \\
\text { ADCP }\end{array}$} & $\mathbf{U V}$ & & - & - & 2.23 & 0.14 \\
\hline & & MV & - & - & - & 2.32 & 0.14 \\
\hline & \multirow{2}{*}{$\begin{array}{l}\text { Standard } \\
\text { NML }\end{array}$} & $\mathbf{U V}$ & 200 & 7.80 & 0.50 & 8.07 & 0.5 \\
\hline & & MV & 200 & 7.68 & 0.49 & 7.92 & 0.49 \\
\hline & \multirow{2}{*}{$\begin{array}{l}\text { Stepped } \\
\text { NML }\end{array}$} & $\mathbf{U V}$ & 200 & (3.74) 7.80 & $(0.24) 0.5$ & $(5.05) 8.09$ & $(0.31) 0.5$ \\
\hline & & MV & 200 & 7.62 & 0.49 & 7.89 & 0.48 \\
\hline & \multicolumn{2}{|c|}{ CHERESKIN } & 200 & 3.65 & 0.24 & 5.14 & 0.31 \\
\hline & \multicolumn{2}{|c|}{ RUDNICK } & 200 & 4.12 & 0.26 & 5.38 & 0.34 \\
\hline \multirow{8}{*}{ 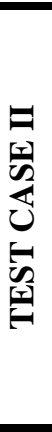 } & \multirow{2}{*}{$\begin{array}{l}\text { OSI- } \\
\text { ADCP }\end{array}$} & $\mathbf{U V}$ & - & - & - & 6.29 & 0.39 \\
\hline & & $\begin{array}{l}\text { MV } \\
0.1 \\
\end{array}$ & - & - & - & 5.92 & 0.36 \\
\hline & \multirow{2}{*}{$\begin{array}{l}\text { Standard } \\
\text { NML }\end{array}$} & UV & 200 & 7.80 & 0.50 & 8.07 & 0.5 \\
\hline & & MV & 200 & 8.10 & 0.52 & 8.41 & 0.52 \\
\hline & \multirow{2}{*}{$\begin{array}{l}\text { Stepped } \\
\text { NML }\end{array}$} & UV & 200 & (3.74) 7.80 & $(0.24) 0.5$ & (5.05) 8.09 & $(0.31) 0.5$ \\
\hline & & MV & 200 & 7.92 & 0.51 & 8.23 & 0.51 \\
\hline & \multicolumn{2}{|c|}{ CHERESKIN } & 200 & 8.78 & 0.56 & 9.76 & 0.6 \\
\hline & \multicolumn{2}{|c|}{ RUDNICK } & 200 & 4.54 & 0.29 & 5.81 & 0.36 \\
\hline \multirow{8}{*}{ 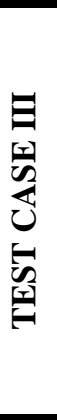 } & \multirow{2}{*}{$\begin{array}{l}\text { OSI } \\
\text { ADCP }\end{array}$} & $\mathbf{U V}$ & - & - & - & 13.08 & 0.82 \\
\hline & & MV & - & - & - & 11.00 & 0.68 \\
\hline & \multirow{2}{*}{$\begin{array}{l}\text { Standard } \\
\text { NML }\end{array}$} & $\mathbf{U V}$ & 200 & 10.42 & 0.67 & 11.14 & 0.69 \\
\hline & & MV & 200 & 10.40 & 0.67 & 11.10 & 0.68 \\
\hline & \multirow{2}{*}{$\begin{array}{l}\text { Stepped } \\
\text { NML }\end{array}$} & UV & (1000) 200 & (8.62) 10.31 & $(0.55) 0.66$ & $(10.04) 11.06$ & $0.62) 0.68$ \\
\hline & & MV & 200 & 10.27 & 0.66 & 11.01 & 0.68 \\
\hline & \multicolumn{2}{|c|}{ CHERESKIN } & 200 & 8.67 & 0.55 & 10.16 & 0.68 \\
\hline & \multicolumn{2}{|c|}{ RUDNICK } & 200 & 8.44 & 0.54 & 9.99 & 0.61 \\
\hline \multirow{8}{*}{ 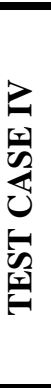 } & \multirow{2}{*}{$\begin{array}{l}\text { OSI } \\
\text { ADCP }\end{array}$} & $\mathbf{U V}$ & - & - & - & 3.79 & 0.25 \\
\hline & & MV & - & - & - & 3.98 & 0.27 \\
\hline & Standard & $\mathbf{U V}$ & 80 & 6.85 & 0.55 & 12.72 & 0.84 \\
\hline & NML & MV & 80 & 6.03 & 0.48 & 12.57 & 0.83 \\
\hline & Stepped & $\mathbf{U V}$ & 200 & 5.18 & 0.41 & 12.16 & 0.81 \\
\hline & NML & MV & 200 & 4.97 & 0.4 & 12.45 & 0.82 \\
\hline & CHERESK & & 80 & 6.97 & 0.56 & 14.72 & 0.98 \\
\hline & RUDNICK & & 80 & 6.78 & 0.54 & 11.07 & 0.73 \\
\hline & OSI & $\mathbf{U V}$ & - & - & - & 3.35 & 0.08 \\
\hline & ADCP & MV & - & - & - & 3.42 & 0.08 \\
\hline 空 & Standard & UV & 200 & 29.5 & 0.83 & 35.22 & 0.86 \\
\hline$\sum_{0}^{\infty}$ & NML & MV & 200 & 29.4 & 0.83 & 35.09 & 0.85 \\
\hline$=$ & Stepped & $\mathbf{U V}$ & 200 & (17.46) 29.13 & $\begin{array}{l}(0.49) 0.82 \\
\end{array}$ & $(20.95) 34.86$ & $(0.51) 0.85$ \\
\hline s & NML & MV & 200 & 29.14 & 0.82 & 34.87 & 0.85 \\
\hline & CHERESK & & 200 & 6.28 & 0.17 & 4.64 & 0.11 \\
\hline & RUDNICK & & 200 & 5.61 & 0.15 & 3.93 & 0.09 \\
\hline
\end{tabular}




\section{Figure 1}

Click here to download Figure(s): figure1.pdf

a)

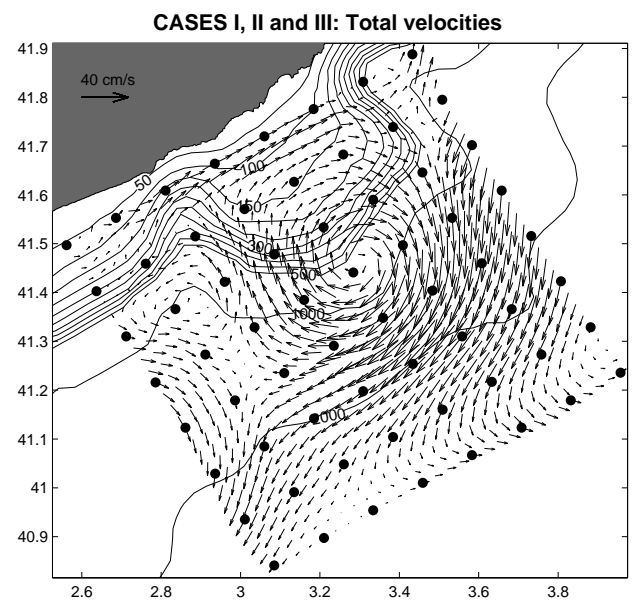

b)

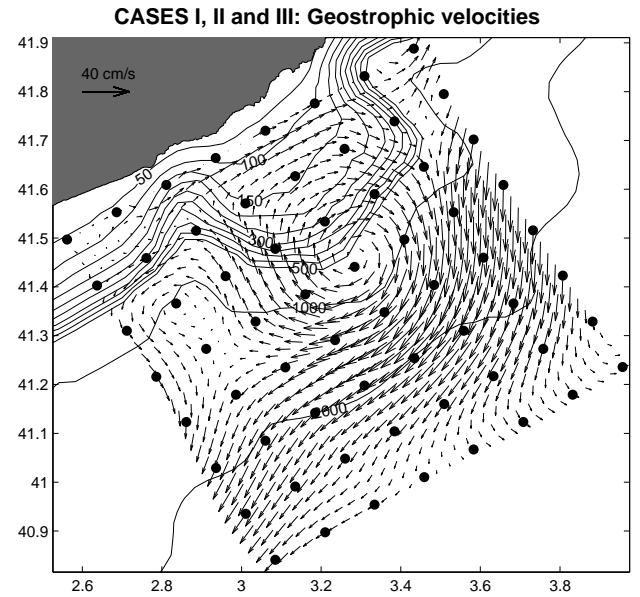

c)

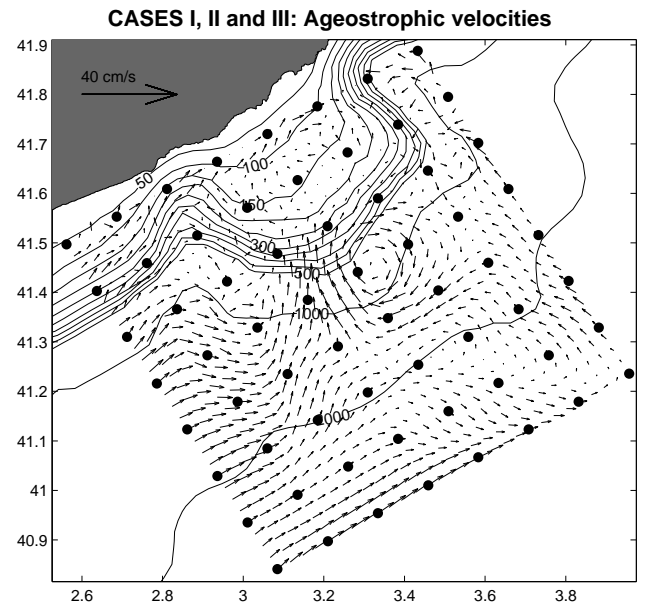

FIGURE 1 


\section{Figure 2}

Click here to download Figure(s): figure2.pdf

a)

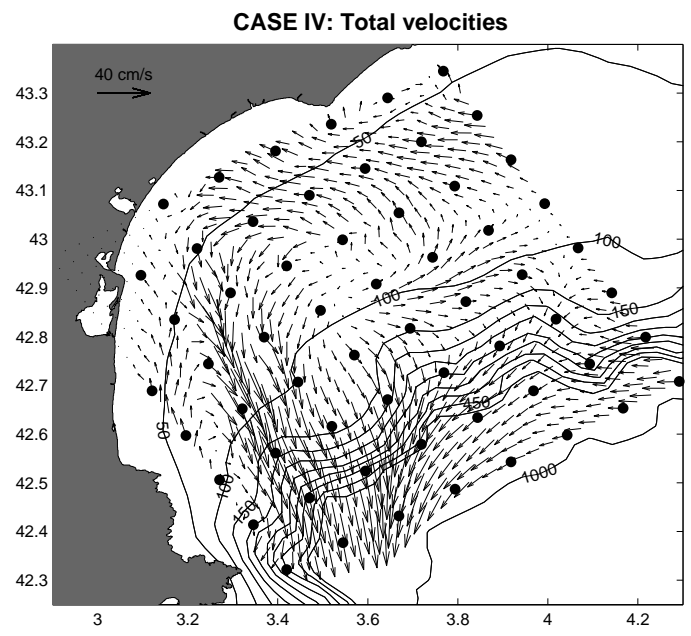

b)

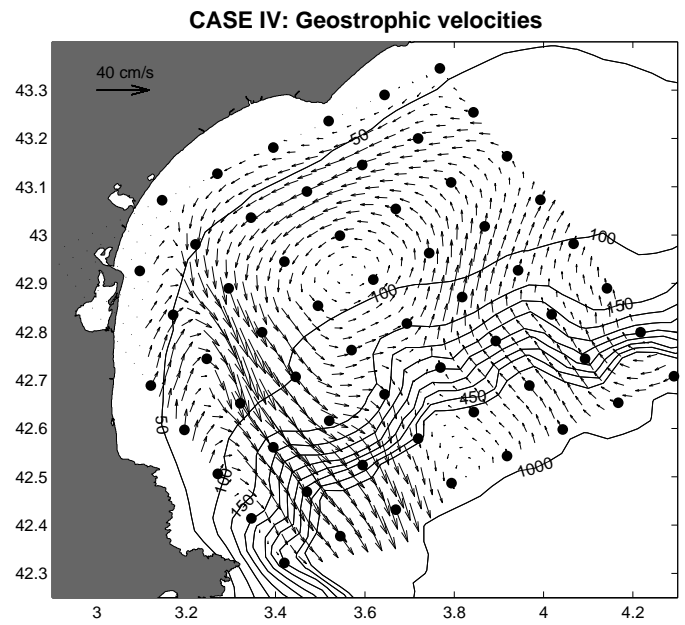

c)

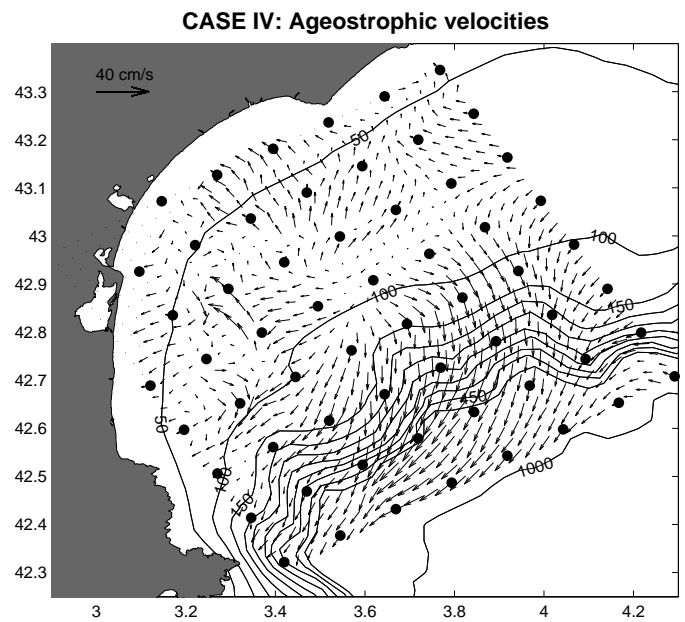

FIGURE 2 


\section{Figure 3}

Click here to download Figure(s): figure3.pdf

a)

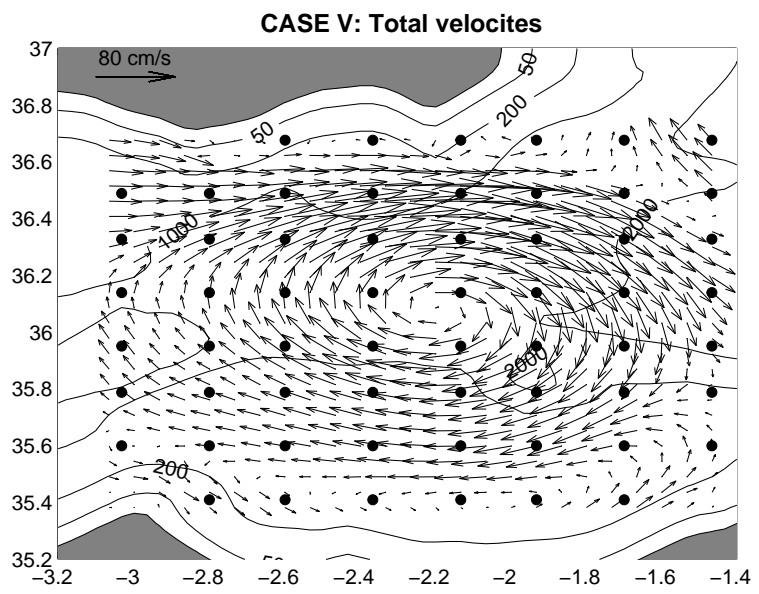

b)

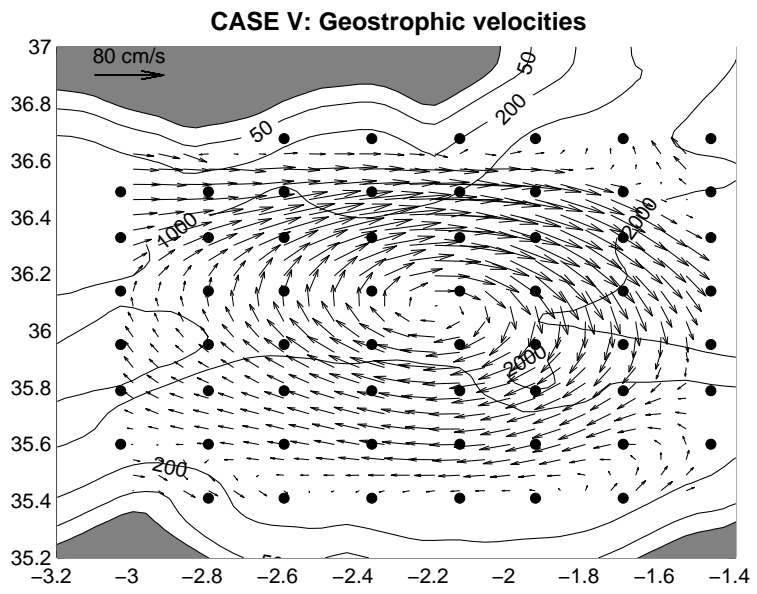

c)

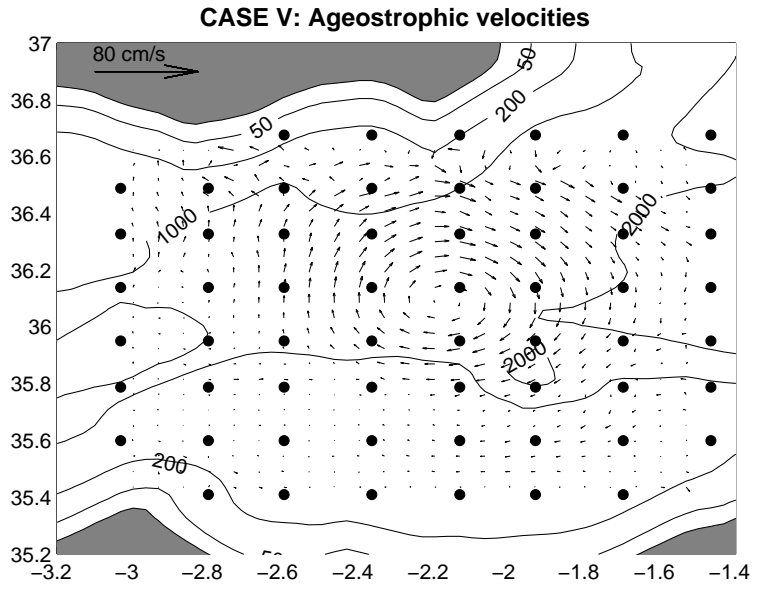

FIGURE 3 

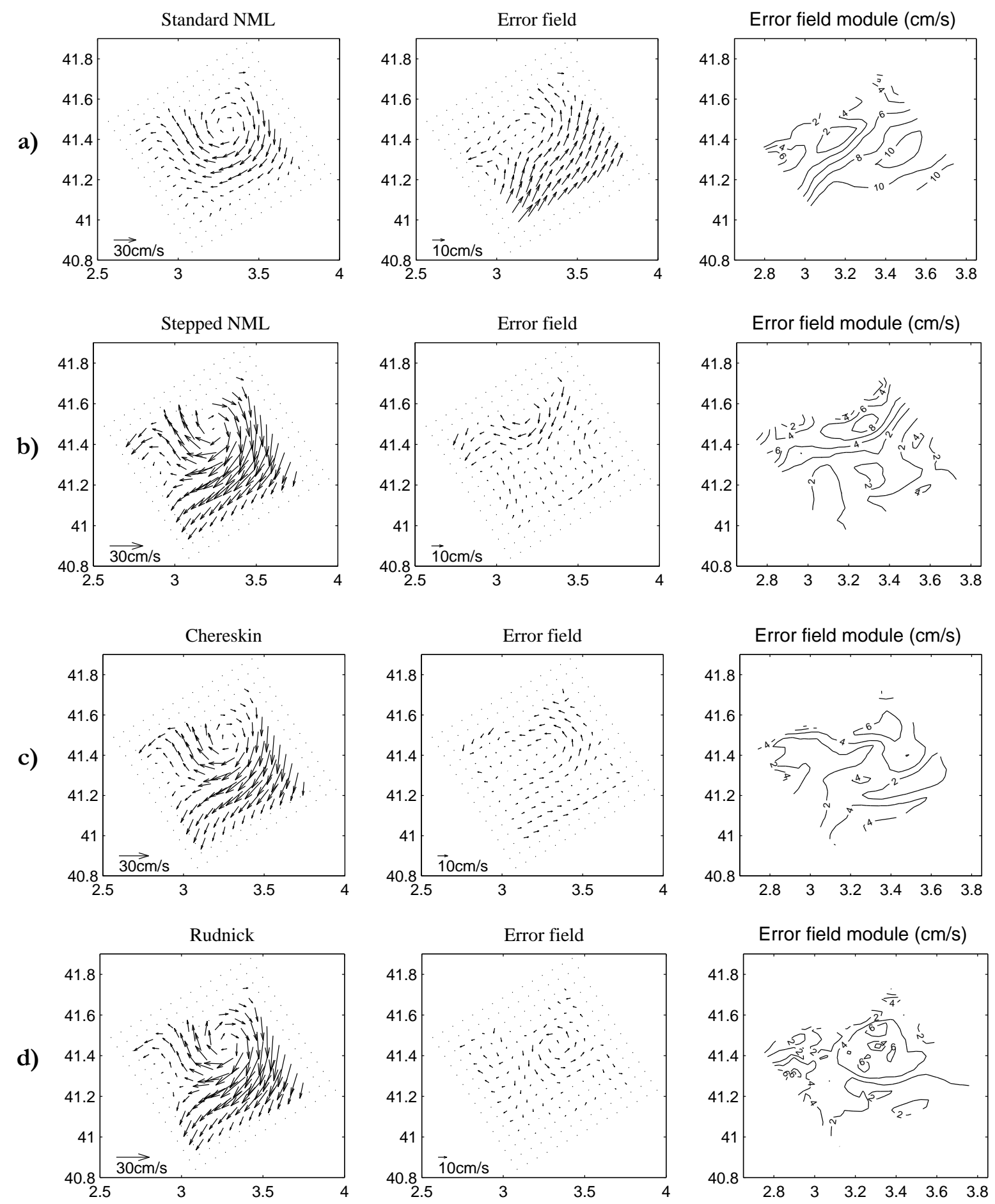

FIGURE 4 
a)

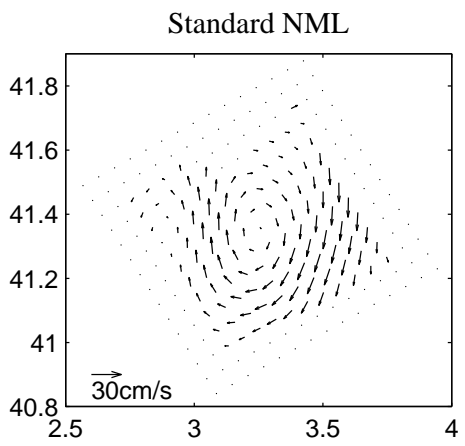

b)

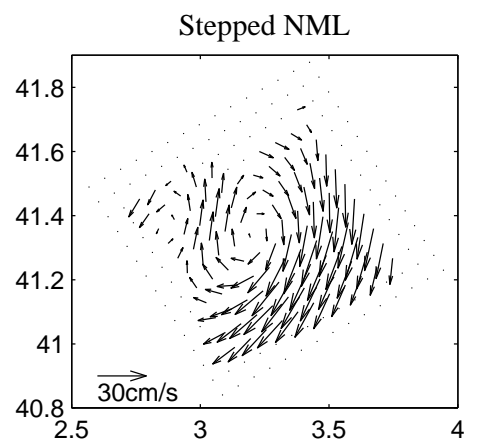

c)

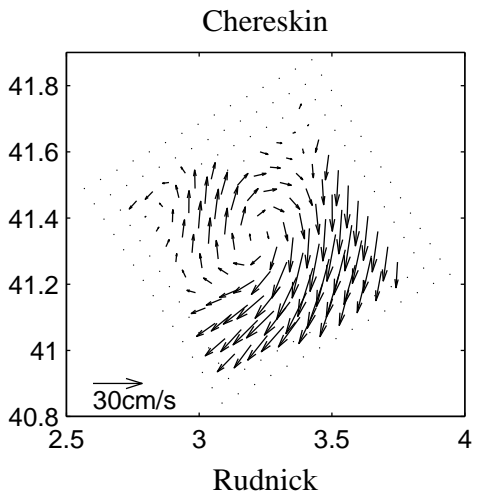

d)

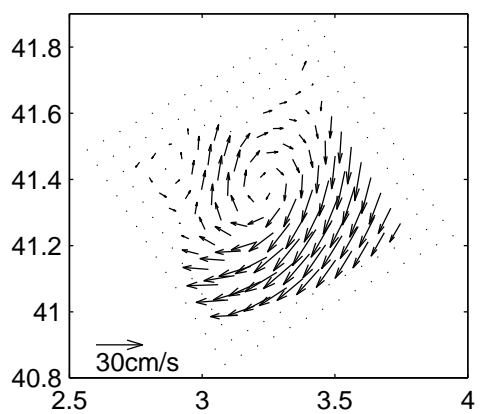

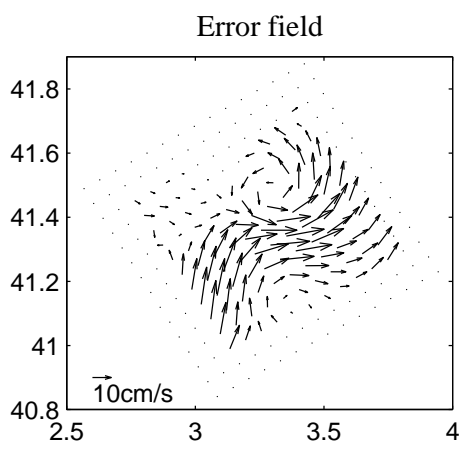
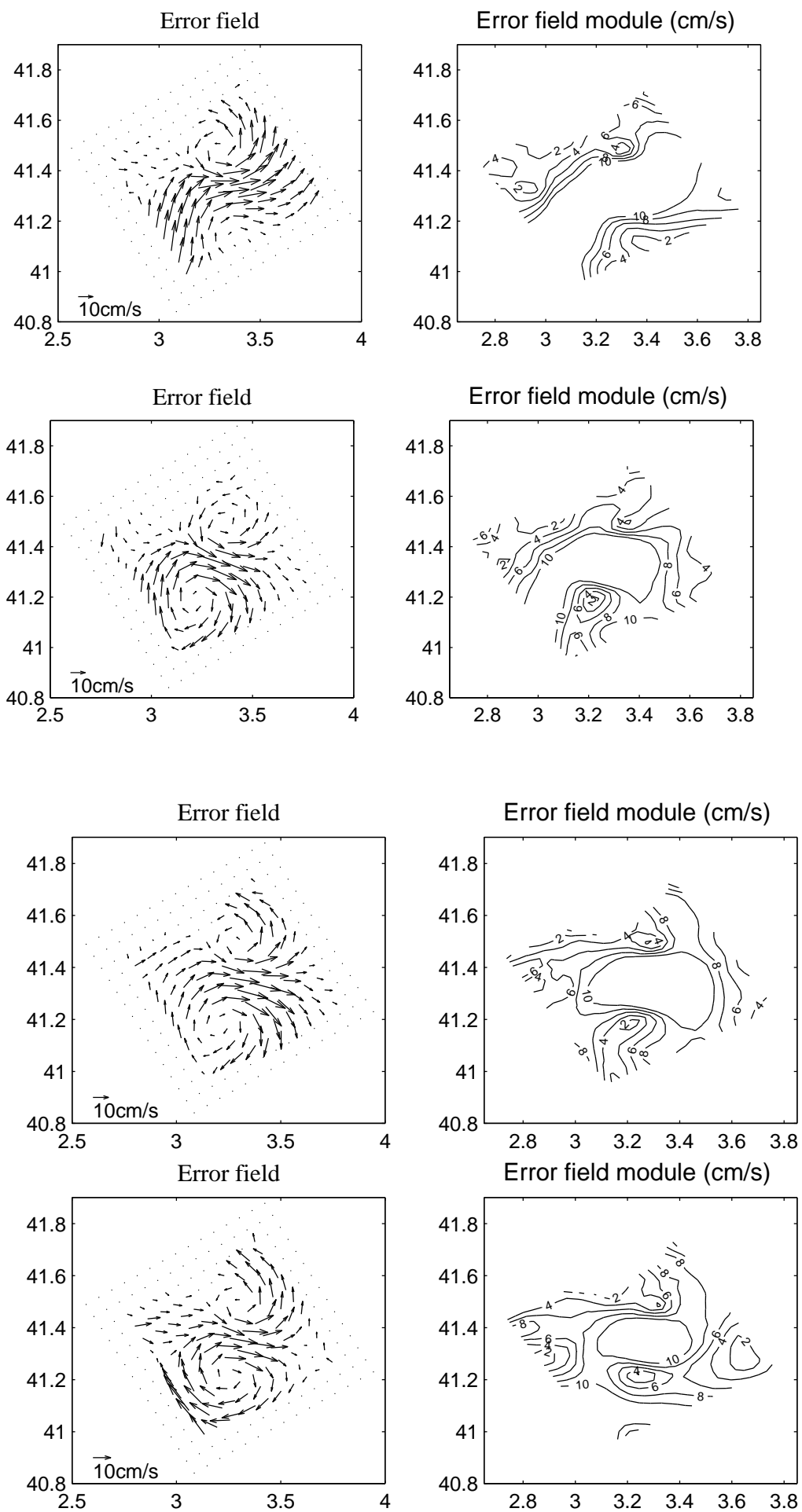

FIGURE 5 
a)

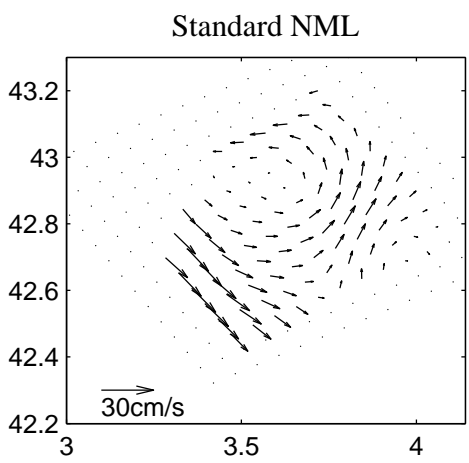

b)

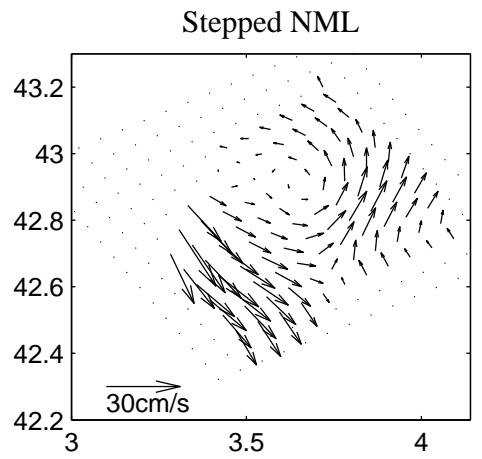

c)

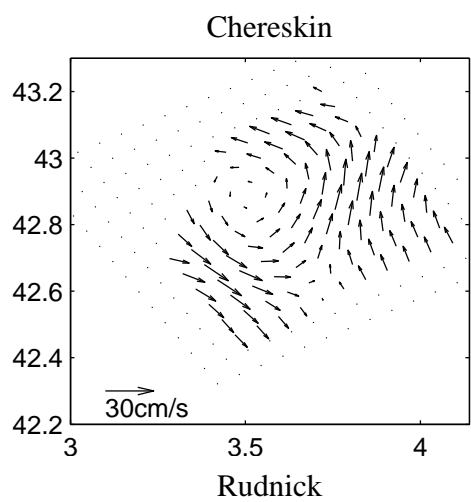

d)

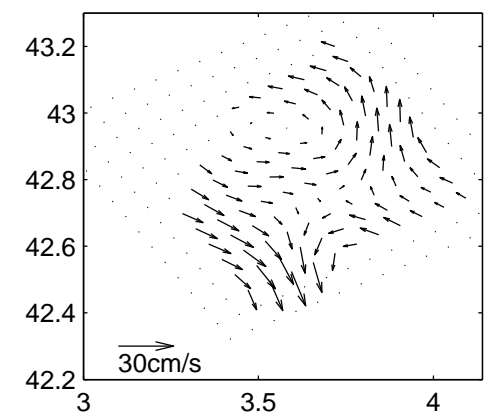

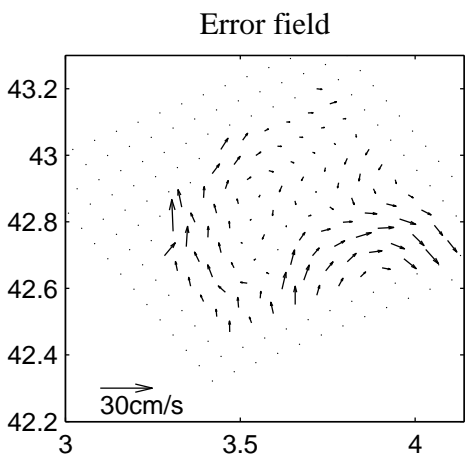
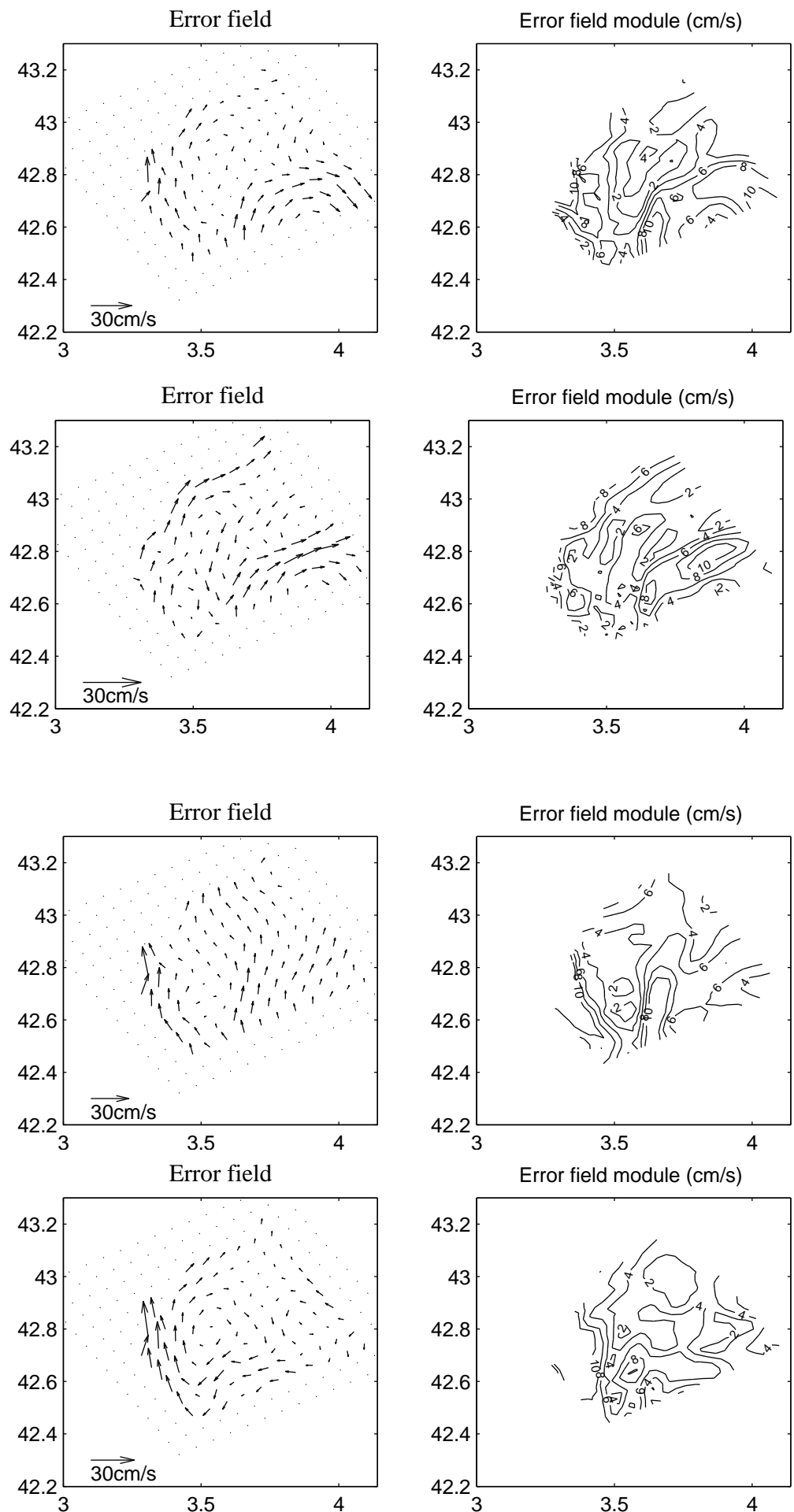

FIGURE 6 
a)

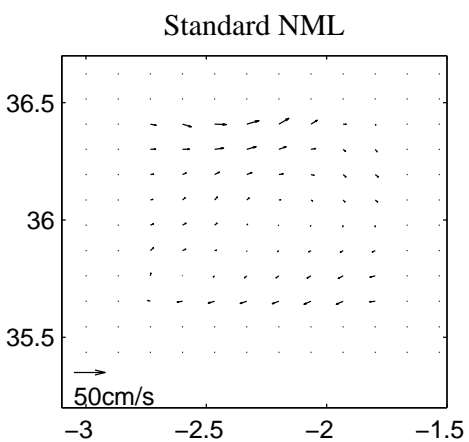

b)

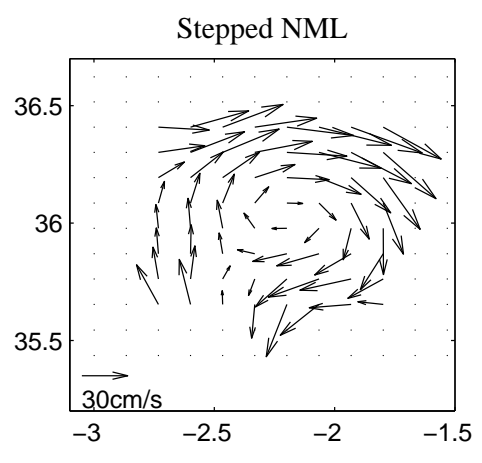

Chereskin

c)

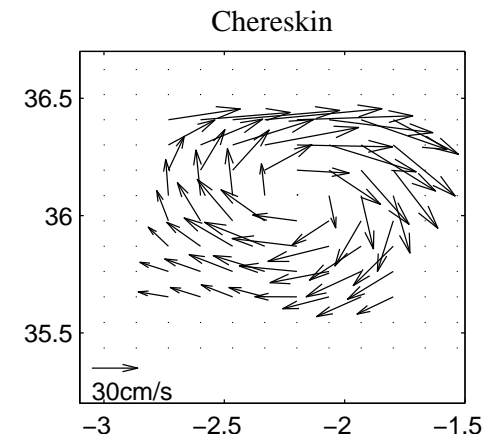

d)

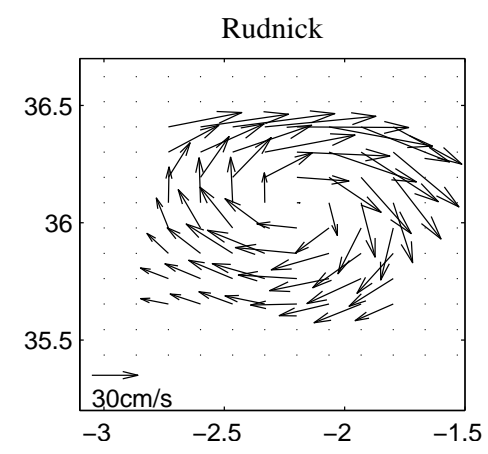

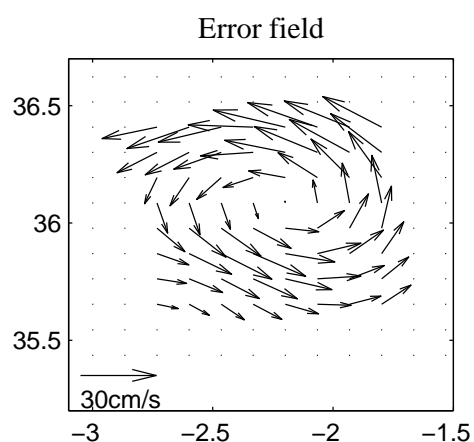
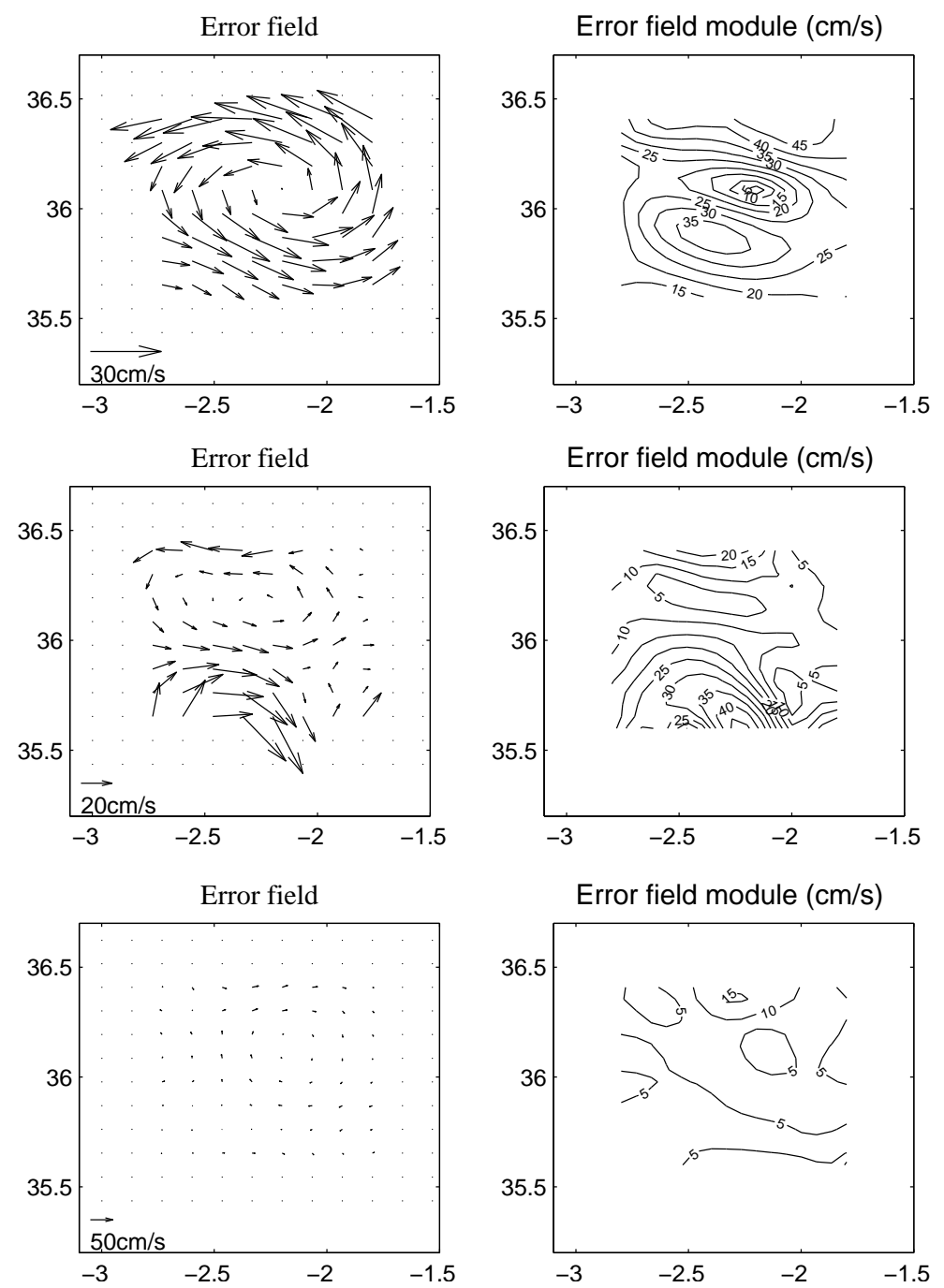

Error field module $(\mathrm{cm} / \mathrm{s})$
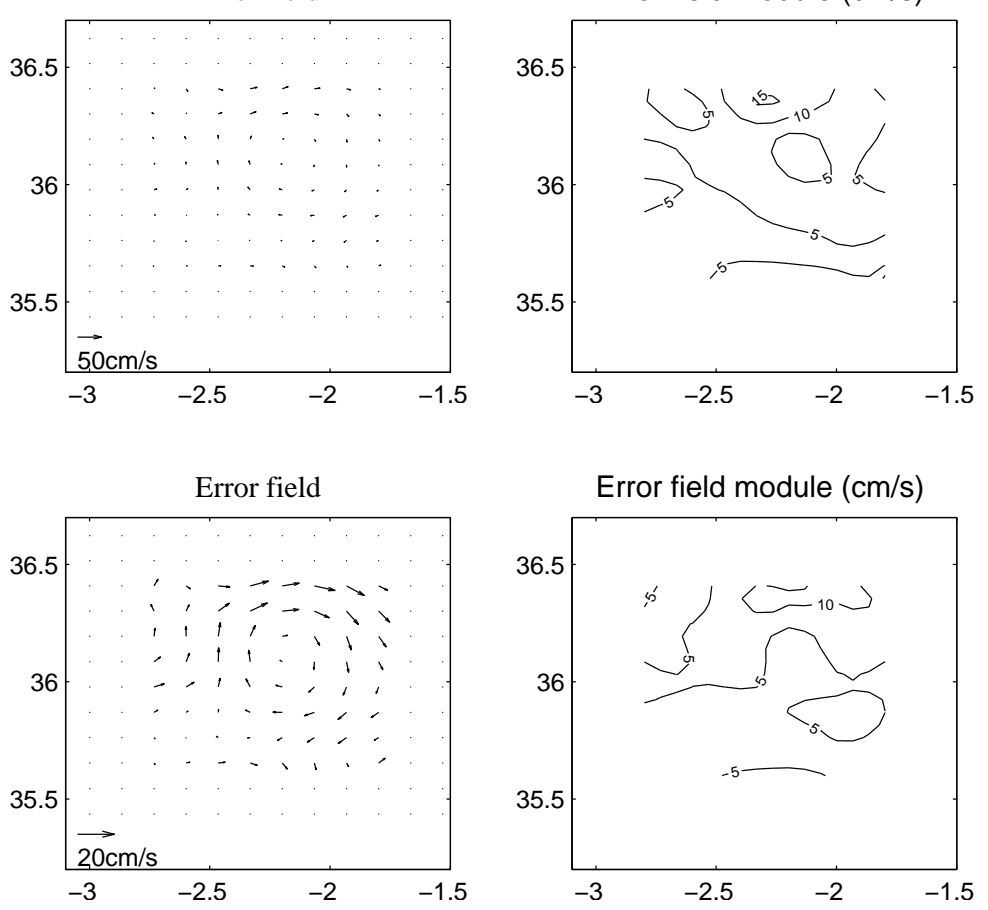

FIGURE 7 


\section{Figure 8}

Click here to download Figure(s): figure8.pdf
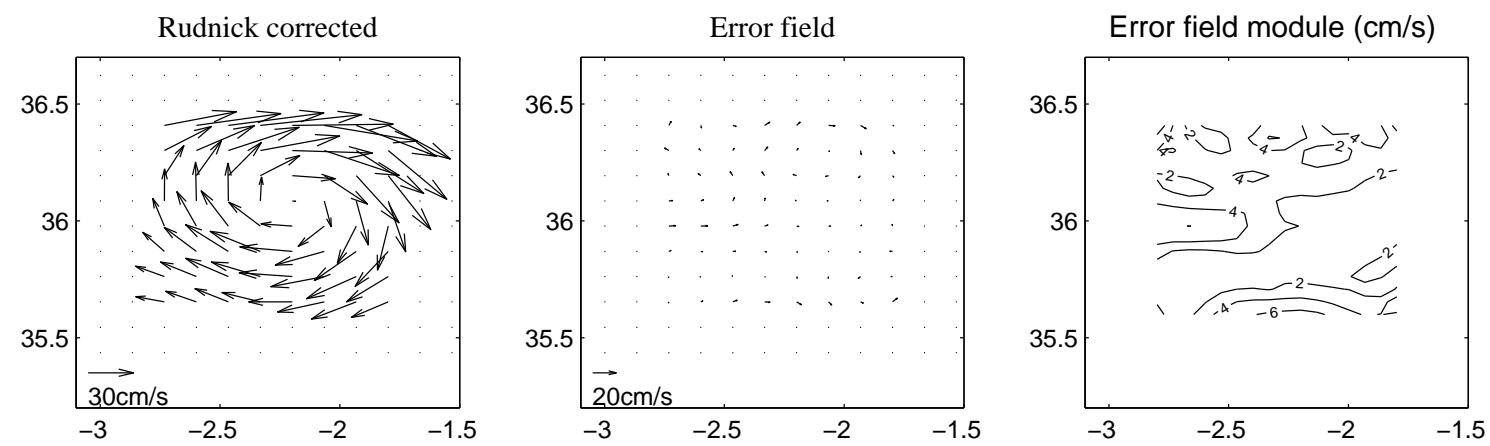

\section{FIGURE 8}

